\title{
Structure and mechanism of TRAPPIII-mediated Rab1 activation
}

Aaron M.N. Joiner ${ }^{1}$, Ben P. Phillips ${ }^{2}$, Kumar Yugandhar ${ }^{3}$, Ethan J. Sanford ${ }^{1}$, Marcus B. Smolka ${ }^{1}$, Haiyuan $\mathrm{Yu}^{3}$, Elizabeth A. Miller², J. Christopher Fromme ${ }^{1,4}$

${ }^{1}$ Department of Molecular Biology and Genetics/Weill Institute for Cell and Molecular Biology, Cornell University, Ithaca, NY, USA

2 MRC Laboratory of Molecular Biology, Cambridge, United Kingdom

${ }^{3}$ Department of Computational Biology/Weill Institute for Cell and Molecular Biology, Cornell University, Ithaca, NY, USA

${ }^{4}$ Corresponding author: jcf14@cornell.edu

\section{ABSTRACT}

The GTPase Rab1 is a master regulator of both the early secretory pathway and autophagy. Rab1 activation is controlled by its GEF (guanine nucleotide exchange factor), the multi-subunit TRAPPIII complex. The Trs85 regulatory subunit is critical for robust activation of Rab1 but its mechanistic role within the complex has remained unclear. Here we report the cryo-EM structure of the intact yeast TRAPPIII complex bound to its substrate Rab1/Ypt1. The orientation of the Rab1/Ypt1 hypervariable domain when bound to the complex leads to a model for how TRAPPIII associates with and activates Rab1/Ypt1 at the membrane surface. We identify a conserved amphipathic $\alpha$-helix motif within Trs85 and demonstrate that this helix is required for stable membrane binding and Rab1/Ypt1 activation by TRAPPIII. Taken together, our results provide a comprehensive analysis of the structure and function of the yeast TRAPPIII complex and reveal that the key function of Trs85 is to serve as a membrane anchor, via its amphipathic helix, for the entire TRAPPIII complex. 


\section{INTRODUCTION}

The GTPase Rab1 is a master regulator of both the early secretory pathway and autophagy (Jedd et al., 1995; Lynch-Day et al., 2010; Zoppino et al., 2010; Hutagalung and Novick, 2011). In budding yeast the Rab1 protein is encoded by the YPT1 gene (Segev, 1991; Segev et al., 1988). Once activated by GTP-binding, Rab1/Ypt1 recruits vesicle tethering factors such as Uso1, Atg11, and the COG complex to initiate membrane fusion events (Jedd et al., 1995; Yamakawa et al., 1996; Cao et al., 1998; Suvorova et al., 2002; Lipatova et al., 2012). Proper localization of Rab1/Ypt1 is directly linked to its activation state and due to a slow intrinsic rate of exchange, the key regulatory step of Rab1/Ypt1 activation is catalyzed by its GEF, the multi-subunit TRAPPIII complex.

In the late secretory pathway, TRAPPII (a distinct, but related multisubunit complex) activates the small GTPase Rab11 (Jedd et al., 1995; Jones et al., 2000; Thomas and Fromme, 2016; Thomas et al., 2019). While the TRAPPII and TRAPPIII complexes share a core set of subunits, the distinguishing feature of the TRAPPIII complex is the presence of the Trs85 subunit (TRAPPC8 in metazoans) (Sacher et al., 1998, 2000, 2001, 2019; Lynch-Day et al., 2010). The metazoan TRAPPIII complex possesses three additional subunits that are not present in budding yeast (Bassik et al., 2013; Riedel et al., 2018) (Table S1), but the 7 genes encoding the 8 subunits of the yeast TRAPPIII complex are highly homologous with their metazoan paralogs (Koumandou et al., 2007; Sacher et al., 2019).

Loss of Trs85 results in a major reduction of Rab1/Ypt1 activation in vivo and in vitro, and causes defects in autophagy and aberrant secretion. TRAPPIII has been 
reported to function at many cellular locations important for secretion and autophagy, including COPII vesicles, the Golgi complex, Atg9 vesicles, and the preautophagosomal structure in both budding yeast and metazoans (Meiling-Wesse et al., 2005; Nazarko et al., 2005; Lynch-Day et al., 2010; Kakuta et al., 2012; Lipatova et al., 2012; Bassik et al., 2013; Tan et al., 2013; Imai et al., 2016; Lamb et al., 2016; Zhao et al., 2017; Thomas et al., 2018; Stanga et al., 2019; Zhang et al., 2020).

Previous reports have presented crystal structures of the TRAPP core subunits and negative-stain electron microscopy analysis of the TRAPPIII complex, which provided an important foundation for understanding the overall architecture and function of the entire TRAPPIII complex (Jang et al., 2002; Kim et al., 2005, 2006; Tan et al., 2013). The nucleotide exchange mechanism was also elucidated using the core subunits bound to Rab1/Ypt1, and the active site was determined to be comprised of small parts of four subunits (Bet5, Bet3, Trs31, and Trs23) (Kim et al., 2006; Cai et al., 2008). However, the mechanistic role of Trs85 in the TRAPPIII complex has remained unclear, the structure of Trs85 (and the entire TRAPPIII complex) has not been determined, and how the complex interacts with membranes in order to activate Rab1/Ypt1 is unknown.

In order to define the role of the Trs85 subunit within the TRAPPIII complex, we have used a comprehensive approach combining cryo-EM, cross-linking massspectrometry, biochemical reconstitution of membrane-binding and nucleotide exchange activity, and in vivo functional analysis. Here we report the high-resolution structure of the intact yeast TRAPPIII complex bound to its substrate GTPase Rab1/Ypt1, representing the key intermediate of the nucleotide exchange reaction. The structure 
reveals that Trs85 possesses both an N-terminal GTPase-like fold and a C-terminal $\alpha$ solenoid motif. The $\alpha$-solenoid connects Trs85 to the TRAPP core, interacting with a core residue mutated in the human disease SEDT. A portion of the flexible "HVD" region of Rab1/Ypt1, which links the GTPase to the membrane, is visible in the structure bound to the Trs31 core subunit. The positioning of the HVD and the electrostatics of the complex suggest a specific orientation of the complex on the membrane surface. We identify a conserved amphipathic $\alpha$-helix motif on the putative membrane-binding surface of Trs85 and determine that this motif is required for stable membrane binding and Rab1/Ypt1 activation both in vitro and in vivo. Our results indicate that this region is also required for the function of Trs85 in autophagy, indicating that TRAPPIII uses the same membrane-binding mechanism to activate Rab1/Ypt1 for both secretion and autophagy. 


\section{RESULTS}

\section{Trs85 anchors the TRAPPIII complex to the membrane surface}

We previously reported that the Trs85 subunit is required for robust Rab1/Ypt1 activation at the Golgi complex in vivo and on synthetic liposomes in vitro (Thomas et al., 2018) (Figure 1A, B). In contrast, Trs85 was dispensable for GEF activity in the absence of membranes (Thomas et al., 2018) (Figure 1C). We hypothesized that a primary function of Trs85 might be to directly interact with the membrane surface. Although we were unable to purify the isolated Trs85 subunit, we compared membrane binding of the purified TRAPPIII complex with and without the Trs85 subunit, using an in vitro liposome pelleting assay (Figure 1D). Consistent with our hypothesis, the intact TRAPPIII complex exhibited robust binding to synthetic liposomes, and the Trs85 subunit was required for stable membrane association (Figure 1E).

\section{Cryo-EM structure of the yeast TRAPPIII-Rab1/Ypt1 complex}

To establish the mechanism of Trs85 function within the complex, we sought to resolve the high-resolution structure of the intact budding yeast (S. cerevisiae) TRAPPIII complex. Furthermore, the published crystal structure of TRAPP core subunits bound to the Rab1/Ypt1 substrate lacks the critical "HVD" (hyper-variable domain) of Rab1/Ypt1 (Cai et al., 2008), which has been shown to play an important role in substrate recognition by the TRAPP complexes (Thomas et al., 2019). We therefore assembled a stable complex in which TRAPPIII was bound to full-length yeast Rab1/Ypt1 in order to also visualize any interactions involving the HVD (Figure S1A). For this purpose, we 
used a yeast Rab1/Ypt1 construct in which the two C-terminal cysteine residues that are prenylated in vivo were replaced with an affinity tag for purification.

This complex was well-behaved and suitable for high-resolution cryo-EM experiments (Figure S2), but the complex exhibited a strongly "preferred orientation" on frozen grids. Initial datasets indicated that approximately $95 \%$ of particles adopted a single orientation, and the other $5 \%$ adopted an orientation that was only slightly different from the primary orientation. To capture additional views, we therefore imaged grids tilted by $30^{\circ}$. Although this resulted in a marked improvement, significant orientation bias remained that precluded confident de novo building of Trs85 into resulting reconstructions. We therefore collected additional data using grids tilted at $40^{\circ}$ and $45^{\circ}$. Although these data were of lower quality due to the higher tilt angle, combination of data from the three tilt angles provided sufficient orientation coverage to generate a $3.7 \AA$ reconstruction suitable for de novo model building (Figure $2 \mathrm{~A}, \mathrm{~B}$, Table S2, Figure S3). For the core subunits and GTPase substrate, we docked and rebuilt available crystal structures of subunits from yeast and other species (Jang et al., 2002; Rak et al., 2003; Kim et al., 2005, 2006; Pylypenko et al., 2006; Cai et al., 2008). For Trs85, we used trRosetta (Yang et al., 2020) to generate models of Trs85 from budding yeast and several other organisms. The overall topologies of these models were quite similar and matched the 3D-reconstruction reasonably well. The trRosetta model of budding yeast Trs85 was therefore used as a guide for de novo building of the Trs85 model. 
The TRAPPIII complex adopts a narrow rod-like structure (Figure 2A, B), in agreement with the published negative-stain EM structure (Tan et al., 2013). The structures of each of the core subunits and the GTPase substrate are largely similar to the published crystal structures (Figure S4). Some small differences were noted, for example, we observed covalently-bound palmitate molecules in both Bet3 subunits, whereas only one of the Bet3 subunits appeared to be bound to palmitate in the previous structure of the catalytic core (Cai et al., 2008). Rather than mediate interactions with organelle membranes like the prenyl groups of Rab1/Ypt1, these palmitates are thought to stabilize the folding of the Bet3 subunits (Turnbull et al., 2005; Kümmel et al., 2006). The interaction between the TRAPP active site and the globular domain of Rab1/Ypt1 is essentially the same as that observed in the crystal structure previously determined by the Reinisch group (Cai et al., 2008). However, we observed a novel interaction with the Rab1/Ypt1 HVD that we discuss further below.

To validate the final structural model, we utilized cross-linking mass spectrometry (XL-MS) to identify lysine residues in proximity to each other (Figure 2C, D, Figure S1B, Table S3). From 108 total cross-links, 32 could be mapped to residues modeled in the structure (Figure 2E). 31 of these 32 cross-links were within the maximum distance constraint for disuccinimidyl sulfoxide (DSSO), 30Å, with most occurring between residues less than $20 \AA$ apart (Figure 2F). The other cross-link (between Lys78 of Trs31 and Lys 85 of Bet3) spanned a distance of $45 \AA$ and is likely a false positive because those two cross-linked residues were also far apart in the crystal structure of these subunits bound to Rab1/Ypt1 (Cai et al., 2008). However, we cannot rule out the possibility that this cross-link might have captured some of the dynamic movement of 
the complex in solution. The remaining 76 cross-links could not be mapped onto the model because they involved one or more residues located in unmodeled loops (Table S3). For each of these residues, we confirmed that the position of these unstructured loops in the model was consistent with the existence of a cross-link. Overall, the XL-MS results contribute support for the atomic model of the complex.

\section{The C-terminus of Trs85 binds to the core subunits Trs 20 and Trs 31}

The cryo-EM structure reveals that the $\mathrm{N}$-terminal portion of Trs85 adopts a GTPase-like fold and the C-terminal portion folds into a twisting $\alpha$-solenoid structure comprising five pairs of $\alpha$-helices (Figure 3A). The $\alpha$-solenoid connects Trs 85 to the TRAPP core through a surface that is well-conserved among Trs85 homologs in related species, and even throughout model organisms and in humans (Figure 3B). This interaction surface is described further below. The GTPase-like fold in Trs85 has multiple insertions relative to a typical GTPase protein, and does not appear capable of binding nucleotide, as key nucleotide-binding residues are not conserved and an $\alpha$-helix formed by residues $46-55$ of Trs 85 occupies the region where nucleotide is bound in GTPase proteins.

The structure indicates that the C-terminal $\alpha$-solenoid region of Trs85 interacts with the TRAPP core. We determined that the C-terminal 198 amino acids of Trs85 (Trs85 ${ }^{501-698}$ ) are sufficient to maintain this interaction during purification of the recombinant complex (Figure 4A, B), and several cross-links were detected between the C-terminal region of Trs85 and either Trs20 or Trs31 in the XL-MS experiments (Figure 2D, E Figure 4D, Table S3). 
There are two interfaces between Trs85 and the TRAPP core subunits: an extensive interaction between Trs85 and Trs20 (Figure 4E), involving 31 residues and encompassing a total surface area of roughly $2,250 \AA$, and a much smaller interaction between Trs31 and part of a largely unstructured loop of Trs85. This loop spans residues $\sim 580-612$ of Trs85, and appears to be an insertion specific to S. cerevisiae.

The interface between Trs85 and Trs20 is quite conserved (Figure 3B), and involves Trs20 Asp46, a residue known to be important for assembly of both the TRAPPII and TRAPPIII complexes (Figure 4E) (Zong et al., 2011; Brunet et al., 2013; Taussig et al., 2014) that is mutated in spondidoepiphyseal dyplasia tarda (SEDT) (Sacher et al., 2019). This and nearby negatively charged residues of Trs20: Glu49, Asp50, and Asp93, interact with multiple positively charged residues of Trs85: Arg618, Arg619, Arg620, and Lys621 (Figure 4E). To test the importance of this interface in vivo, we generated alanine substitution and charge reversal mutations for the Trs 85 residues and assessed their ability to provide Trs85 function in a sensitized background in which the core Bet3 subunit is tagged at its C-terminus (Sacher et al., 2001). All of the mutants dramatically impaired Trs85 function (Figure 4F). Even mutation of just one of these residues, Trs85 Arg620, to either alanine or glutamate, significantly impaired Trs85 function, with R620E exhibiting a stronger phenotype than R620A (Figure 4F).

To determine whether these phenotypes were due to disruption of the Trs85Trs20 interaction, we investigated the assembly status of the TRAPPIII complex by monitoring the amount of the core subunit Trs23 that co-immunoprecipitated with the Trs85 mutant alleles (Figure 4G). The quadruple mutants and R620E single mutant essentially abolished the interaction between Trs85 and the core subunits, whereas the 
R620A substitution mutant exhibited a partial effect. As the severity of complex disruption correlated with the growth phenotypes in the complementation test, these results provide a strong validation of the importance of the Trs85-Trs20 interface observed in the cryo-EM structure (Figure 4F, G).

We previously reported that Trs85 is required for robust activation of Rab1/Ypt1 at the Golgi complex (Thomas et al., 2018). We tested the ability of these complexdisrupting mutants to activate Rab1/Ypt1 at the Golgi in otherwise wild-type cells (Figure $4 \mathrm{H})$. As predicted, the quadruple mutants and R620E single mutant were unable to robustly activate Rab1/Ypt1, as monitored by the loss of punctate RFP-Ypt1 localization. The R620A mutant again exhibited an intermediate phenotype. Interestingly, the complex-disrupting mutations also resulted in mislocalization of Trs85 (Figure 4H), suggesting that Trs85 is not able to stably bind to membranes by itself when not incorporated into the TRAPPIII complex.

To examine the importance of the less conserved interface between Trs 85 and Trs31 (Figure 2B), we tested a mutant lacking a portion of the partially structured loop designed to disrupt this interaction without disrupting the folding of Trs85 (Trs85 ${ }^{\Delta 575-603}$ ). This Trs85 mutant exhibited a minor temperature-sensitive growth phenotype and a partial loss of interaction with the TRAPP core in co-IP experiments (Figure 4F, G). To further examine the effect of this mutant, we attempted purification of recombinant TRAPPIII harboring this mutation. The complex remained largely intact during nickel-ion affinity purification but then dissociated during gel filtration chromatography (Figure 4C), suggesting a weakened ability of this Trs85 mutant to stably associate with the core subunits. In contrast, we were unable to purify the quadruple mutant complexes 
disrupting the Trs20 interaction, consistent with an inability to purify the isolated Trs85 subunit. Therefore, the loop comprising residues 575-603 plays a subsidiary role in linking Trs85 to the TRAPP core subunits by interacting with Trs31.

\section{A model for the orientation of TRAPPIII on the membrane surface}

The TRAPP complexes identify their specific substrates in part through recognition of the GTPase HVD (Thomas et al., 2019). It is likely that other Rab-GEFs also recognize the HVD regions of their GTPase substrates (Chavrier et al., 1991; Dunn et al., 1993; Li et al., 2014), but interactions between GEFs and GTPase HVD regions have not been structurally characterized. In fact, HVD regions are often intentionally omitted from structural studies because of their "unstructured" nature (Dong et al., 2007; Cai et al., 2008). In our cryo-EM reconstruction of the TRAPPIII-GTPase complex, we observed a region of strong but unexpected density on the surface of the complex that we have confidently assigned as corresponding to a portion of the Rab1/Ypt1 HVD (Figure 2B and $\mathrm{S} 4 \mathrm{H}$ ). There are multiple pieces of evidence supporting this assertion: 1) At lower threshold levels of the reconstruction, there is a nearly continuous tube of density connecting this region of unknown density with the C-terminus of the Rab1/Ypt1 nucleotide-binding domain (NBD) (Figure 5A); 2) In preliminary cryo-EM experiments, we observed 2D classes corresponding to complexes either with or without bound Rab1/Ypt1, and the unknown density was only present when Rab1/Ypt1 was also bound (Figure 5B); 3) XL-MS data identified cross-links between the Rab1/Ypt1 HVD and the Trs31 subunit, occurring in the region between the C-terminus of the Rab1/Ypt1 globular domain and the unknown density (Figure 5C, D, Table S3). Therefore, although 
the cryo-EM density is not clear enough to unambiguously assign specific residues of Rab1/Ypt1 to this region, we are confident that it corresponds to some portion of the Rab1/Ypt1 C-terminal HVD.

The Rab1/Ypt1 HVD culminates with two cysteine residues that are prenylated (Pylypenko et al., 2006) and insert into the membrane either prior to or concomitant with activation by TRAPPIII. The observed location of the HVD bound to the surface of Trs31 therefore imposes a significant constraint upon any model for how TRAPPIII binds to the membrane surface. This leads us to propose that the complex binds the membrane in the orientation shown in Figure 5A such that the Rab1/Ypt1 NBD is bound to the surface of TRAPPIII that is most distal to the membrane. Additional evidence in favor of this orientation of the TRAPPIII complex arises from analysis of the electrostatic potential of the surface of the complex, though this analysis has the caveat that some surface residues are unstructured. While there are both positive and negative charges scattered across all surfaces of the TRAPPIII complex, and no single surface contains a concentrated region of positive charge, the proposed membrane-binding surface appears to be the most positively charged of all the surfaces (Figure 5E). In accordance with this idea, we determined that the TRAPPIII complex prefers to bind liposomes with anionic lipid mixtures (Figure 5F, Table S4). A circumstantial piece of evidence supporting the proposed membrane-bound orientation is the extreme preferred orientation adopted by the complex in vitreous ice (Figure S2F). These 2D projections (Figures 5B, S2B) implicate two possibilities for which surface was interacting with the air-water interface within the holes of the EM grid: one is opposite where the Rab1/Ypt1 C-terminus is located, while the other is the same surface we propose TRAPPIII uses to 
bind membranes (Figure 5A). As a peripheral membrane protein, we think it is probable that TRAPPIII interacted with the air-water interface via the same surface it uses to bind to organelle membranes within a cell.

\section{A conserved amphipathic helix within Trs85 is required for TRAPPIII membrane binding and Rab1 activation}

We were concerned that the proposed membrane binding surface of Trs 85 did not appear to possess an overwhelmingly positively charged character, which we expected to be necessary for its interaction with membranes. However, upon closer inspection we identified an unmodeled region on this surface located between the GTPase-like fold and $\alpha$-solenoid motif (residues 368-409). Though we were unable to model this portion of the protein in our structure because of a lack of clear electron density, the primary sequence is conserved and predicted to fold as an amphipathic $\alpha$ helix (Figure 6A-C).

In order to determine whether this putative amphipathic helix was important for Trs85 and TRAPPIII function, we made several mutations in this region, disrupting either the charge or both charge and hydrophobicity (Figure 6D). Mutation of 7 residues in this region to alanine (mutant 7A) resulted in severe loss of function (Figure 6E). Removal of the basic charge (mutant 4A) displayed slight growth defects, while charge inversion of the lysine residues by mutation to glutamate residues (mutant $4 \mathrm{E}$ ) resulted in complete loss of function (Figure 6E).

We considered the possibility that these mutations might prevent Trs85 from associating with the core TRAPP subunits, but both the 7A and 4E mutants were stably 
bound to the TRAPP core in co-IP experiments (Figure 6F). We reasoned that if the 7A and 4E Trs85 mutants associate with the core TRAPP subunits but are not functional, then perhaps these residues are indeed part of an amphipathic helix responsible for mediating TRAPPIII membrane-binding, similar to the role of amphipathic helices in many other peripheral membrane proteins (Kahn et al., 1992; Antonny et al., 1997; Huang et al., 2001; Lee et al., 2005; Drin and Antonny, 2010). To test this hypothesis, we purified recombinant mutant TRAPPIII complexes and directly tested their relative membrane-binding affinities using the in vitro liposome pelleting assay (Figure 6G). Similar to wild-type, a small amount of each complex pelleted in the absence of membranes. However, in striking contrast to wild-type, both the 7A and 4E mutants did not bind to synthetic liposomes (Figure $6 \mathrm{H})$, indicating that this amphipathic helix is required for TRAPPIII membrane binding in vitro.

Consistent with a loss of membrane-binding, both the 7A and 4E Trs85 mutants failed to localize to the Golgi and did not activate Rab1/Ypt1 either in vivo (Figure 6I) or in biochemical reconstitution assays in which TRAPPIII GEF activity is membranedependent (Figure 6J). Importantly, these mutants retained their ability to activate Rab1/Ypt1 in a membrane-independent GEF assay (Figure 6K), indicating that the active site is unaffected by these mutations. Taken together, these results reveal that the Trs85 amphipathic helix is required for TRAPPIII complex membrane binding and function.

\section{Similar requirements for TRAPPIII function in secretion and autophagy}


Prior work has shown that trs85 $\Delta$ cells display a deficiency in autophagy (MeilingWesse et al., 2005; Nazarko et al., 2005; Lynch-Day et al., 2010; Kakuta et al., 2012), a defect that can be monitored via the degradation kinetics of GFP-tagged Atg8, the yeast paralog of LC3 (Klionsky et al., 2016). In wild-type cells GFP-Atg8 is degraded as a consequence of rapamycin-induced autophagy, resulting in an accumulation of free GFP. We determined that mutations disrupting the Trs85 amphipathic helix or Trs85 binding to the TRAPP core displayed significant defects in rapamycin-induced GFPAtg8 degradation (Figure 7A, B). This indicates that the role of the Trs85 amphipathic helix in anchoring the TRAPP core to the membrane surface is critical for both secretion and autophagy (Figure 7C). 


\section{DISCUSSION}

GEFs control where and when their GTPase substrates are activated by catalyzing nucleotide-exchange. By coupling a localization signal and nucleotide exchange activity within each GEF, the cell can achieve precise spatial and temporal activation of its substrate GTPase at a specific cellular location. The TRAPP complexes activate two of the most important Rab GTPases in the secretory pathway, Rab1 and Rab11. Recent work from our lab and others has clarified the roles of TRAPPIII and TRAPPII as GEFs for Rab1 and Rab11, respectively (Jones et al., 2000; Meiling-Wesse et al., 2005; Morozova et al., 2006; Lynch-Day et al., 2010; Thomas and Fromme, 2016; Riedel et al., 2018; Thomas et al., 2018, 2019). Although the activation of Rab1/Ypt1 by core TRAPP subunits has been extensively studied (Jones et al., 2000; Wang et al., 2000; Cai et al., 2008), the structure and function of the key TRAPPIII regulatory subunit Trs85 has remained elusive. Our results demonstrate that Trs85 serves as a membrane anchor for the TRAPPIII complex by utilizing a conserved amphipathic helix.

The ability of Trs 85 to anchor the TRAPPIII complex to a membrane surface is vital to the activation of Rab1/Ypt1 because the stable association of the TRAPPIII complex to the membrane surface establishes the proper spatial geometry and likely increases the frequency of interactions between the active site and the Rab1/Ypt1 substrate (Figure 7C). Simply put, the observed elevated activity of TRAPPIII relative to the TRAPP core is due to the fact that the core does not appear able to adequately bind to membranes, and therefore cannot activate Rab1/Ypt1 at the membrane surface.

A key aspect of our work is the presence of the Rab1/Ypt1 HVD in the cryo-EM structure of the TRAPPIII-Rab1/Ypt1 complex. The HVD terminates with two prenylated 
cysteine residues that serve as membrane anchors for the Rab (Pylypenko et al., 2006).

At steady-state in cells, inactive (GDP-bound) Rabs are bound to GDI proteins that protect the hydrophobic prenyl groups (Goody et al., 2005; Wu et al., 2010). However, the inactive Rabs dynamically dissociate from GDI and transiently bind to membranes before re-associating with GDI. In this way, inactive Rabs are thought to sample different organelles before encountering their specific GEF to become activated at a particular organelle. The active Rab cannot bind to GDI and is therefore stabilized at its site of activation until inactivated by a Rab-GAP. Accordingly, the structure that we have determined here represents an intermediate of the nucleotide-exchange reaction in which the prenylated cysteines of the HVD are membrane-bound. This structure reveals the HVD is bound by the Trs31 subunit, and its position in the structure provided us with a critical piece of evidence regarding the orientation of the complex on the membrane surface.

Our identification of the TRAPPIII membrane binding surface led to, and was further supported by, the presence of a conserved amphipathic helix within Trs85. We found that mutations disrupting either the hydrophobic face of the helix or inverting the charge of the other face completely abrogated membrane binding of the entire TRAPPIII complex, both in vitro and in vivo.

The fact that we were not able to model the amphipathic helix provides additional, although perhaps counterintuitive, evidence in favor of its role in membrane binding. Amphipathic helices are very commonly found on the surfaces of proteins, in which they do not perform any specialized function but rather simply are part of the protein fold in which hydrophobic sidechains are on the interior and hydrophilic 
sidechains are on the exterior of the folded protein. These $\alpha$-helices should be readily visible in the structure, as are all of the other predicted $\alpha$-helices in the TRAPPIII complex structure. However, an amphipathic $\alpha$-helix that has evolved to insert into the membrane would not be expected to form part of the hydrophilic surface of a folded protein domain (Drin and Antonny, 2010). Rather, it should extend from the globular portion of the protein in order to insert longitudinally into the outer leaflet of the membrane, as is the case with the Trs85 amphipathic $\alpha$-helix that is required for TRAPPIII membrane binding.

Although there are many examples of amphipathic helices serving as membrane anchors for smaller proteins, such as Arf GTPases and N-BAR domain containing proteins (Drin and Antonny, 2010), TRAPPIII represents a notable example in which a sizeable multi-protein complex is anchored by a single amphipathic helix. Remarkably, the mutation of just four residues in this helix was sufficient to disrupt the binding, and therefore function, of the entire $250 \mathrm{kD}$ complex.

The core subunits likely provide additional protein-protein interactions or perhaps supplementary interactions with organelle membranes, as Trs85 localization to the Golgi was abrogated in cells when mutations that diminished its binding to the core were introduced. We determined that the TRAPPIII complex prefers to bind to membranes with a significant anionic charge, but did not identify a specific lipid requirement (Figure 5F, Table S4). Given that TRAPPIII has been implicated to function at many locations within the cell including on COPII vesicles, at the Golgi, on Atg9 vesicles, and at the preautophagosomal structure, it is likely that additional protein binding partners help specify TRAPPIII localization in vivo via coincidence detection. 
For example, TRAPPIII localization to COPII vesicles likely depends upon an interaction between the TRAPP core and the COPII coat subunit Sec23 (Cai et al., 2007; Lord et al., 2011). Future studies of such functions of TRAPP core subunits are important but made challenging by the need to disentangle observed roles of these subunits in TRAPPIII versus TRAPPII.

Many questions remain regarding the role of both TRAPPIII and TRAPPII in autophagy. Our results indicate that the key function of Trs85, serving as a membrane anchor for the TRAPPIII complex, is important for both normal growth and autophagy (Figure 7C). 


\section{MATERIALS AND METHODS}

\section{TRAPPIII purification}

The full set of TRAPPIII complex genes from Saccharomyces cerevisiae were cloned into two plasmids. Two copies of TRS33 were included to compensate for the otherwise substoichiometric expression levels of this subunit. TRS85 with an $\mathrm{N}$-terminal hexahistidine tag and TRS33 were inserted into a pETDuet-1 vector (CFB2868). As reported previously, BET3, BET5, TRS20, TRS23, TRS31, and TRS33 were inserted into a pCOLADuet-1 vector (pLT21,(Thomas and Fromme, 2016)). These plasmids were co-transformed into the Rosetta2 strain of E.coli (Novagen). Multiple liters of culture were grown in terrific broth for $8-12 \mathrm{~h}$ at $37^{\circ} \mathrm{C}$ (until the $\mathrm{OD}_{600} \sim 2-3$ ). The temperature was then reduced to $16^{\circ} \mathrm{C}$ and 1 hour later IPTG was added to a final concentration of $300 \mu \mathrm{M}$ to induce protein expression. Following overnight expression (14-18h), cells were collected via centrifugation and resuspended in lysis buffer (40mM Tris $\mathrm{pH} 8,300 \mathrm{mM} \mathrm{NaCl}, 10 \%$ glycerol, $10 \mathrm{mM}$ imidazole, $1 \mathrm{mM}$ DTT, 1mM PMSF and $1 \mathrm{x}$ Roche PIC). Cells were lysed by sonication and after clearing the lysate, the complex was purified by nickel affinity chromatography (Ni-NTA resin, Qiagen) and eluted with elution buffer (40mM Tris $\mathrm{pH} 8,300 \mathrm{mM} \mathrm{NaCl}, 10 \%$ glycerol, $250 \mathrm{mM}$ imidazole, $1 \mathrm{mM}$ DTT). The sample was further purified using anion exchange chromatography with a linear gradient on a MonoQ column (GE Healthcare) (Buffer A: 20mM Tris pH 8, 1mM DTT; Buffer B: $20 \mathrm{mM}$ Tris $\mathrm{pH} 8,1 \mathrm{M} \mathrm{NaCl}, 1 \mathrm{mM}$ DTT). The peak fractions were collected, concentrated, and frozen in liquid nitrogen prior to storage at $-80^{\circ} \mathrm{C}$. The fractions were thawed (or never frozen) and concentrated for size exclusion chromatography on a Superdex200 10/300 (GE Healthcare) preequilibrated in 10mM 
Tris $\mathrm{pH} 8,150 \mathrm{mM} \mathrm{NaCl}, 1 \mathrm{mM}$ DTT.

Ypt1 Purification

GST-Ypt1-7xHis (gift from T. Bretscher lab) or GST-Ypt1 (pLT50,(Thomas and Fromme, 2016)), with a cleavable N- terminal GST tag in the pGEX-6P vector backbone, were transformed into the Rosetta2 strain of E.coli (Novagen). 1-4 liters of culture were grown in terrific broth for $8-12 \mathrm{~h}$ at $37^{\circ} \mathrm{C}$ (until the $\mathrm{OD}_{600} \sim 2-3$ ). The temperature was then reduced to $16^{\circ} \mathrm{C}$ and 1 hour later IPTG was added to a final concentration of $300 \mu \mathrm{M}$ to induce protein expression. Following overnight expression (14-18h), cells were collected via centrifugation and resuspended in lysis buffer (1x PBS, $5 \mathrm{mM}$ BME, $2 \mathrm{mM} \mathrm{MgCl} 2$ ). Cells were lysed by sonication and the clarified lysate was incubated with Glutathione resin (G-Biosciences) for $2-3 \mathrm{~h}$ at $4^{\circ} \mathrm{C}$ to isolate GSTtagged proteins. The resin was washed in lysis buffer, resuspended in PreScission cleavage buffer $(50 \mathrm{mM}$ Tris $\mathrm{pH} 7.5,150 \mathrm{mM} \mathrm{NaCl}, 1 \mathrm{mM}$ EDTA, $2 \mathrm{mM} \mathrm{MgCl}$, and $1 \mathrm{mM}$ DTT), and treated overnight with PreScission (3C) protease $(\sim 40 \mathrm{uL}$ at $1.3 \mathrm{mg} / \mathrm{mL})$ at $4^{\circ} \mathrm{C}$ to remove the GST tag. Cleavage of the GST tag eluted the proteins from the resin. After treatment, the supernatant was collected and analyzed by SDS-PAGE.

\section{TRAPPIII-Rab1/Ypt1 complex formation}

Purified TRAPPIII complex (from anionic exchange step) and Ypt1-7xHis were mixed in a $1: 3$ molar ratio and incubated overnight at $4^{\circ} \mathrm{C}$ with calf intestinal alkaline phosphatase (Sigma) to hydrolyze nucleotide. After 12-18h, the sample mixture was subjected to size exclusion chromatography on a Superdex 200 Increase 10/300 column (GE Healthcare) in EM buffer (10mM Tris pH 7.5, 350mM NaCl, 1mM DTT). The 
trace displayed two dominant peaks corresponding to TRAPPIII-Rab1/Ypt1 complex and free Rab1/Ypt1 (Figure S1). The fractions containing the complex were pooled and concentrated in a 100kDa molecular weight cutoff concentrator (Millipore). The protein was then used immediately to prepare grids for cryo-EM.

Cryo-EM sample preparation, data collection, and data processing

The complex, at $0.1-0.3 \mathrm{mg} / \mathrm{mL}$ in EM buffer containing either $0.02 \%$ Tween-20 or 0.025\% amphipol A8-35 (Anatrace) was applied to Quantifoil or UltrAufoil R1.2/1.3 grids (Quantifoil) then blotted for $2 \mathrm{~s}$ at $4^{\circ} \mathrm{C}$ with $100 \%$ humidity and plunged into liquid nitrogen-cooled liquid ethane using a Vitrobot IV (TFS).

\section{“Flat" data:}

Initial imaging data were collected at the MRC-LMB on a Titan Krios operated at $300 \mathrm{kV}$ using a Falcon-III detector and 59kX nominal magnification resulting in a $1.34 \AA /$ pixel size on the detector. A total of 809 fractionated exposures were obtained over two different 24-hour data collection sessions. RELION versions 2, 3, and 3.1 (Scheres, 2012; Kimanius et al., 2016; Zivanov et al., 2018, 2020) were used for all data processing steps. MotionCor2 (Zheng et al., 2017) was used to perform motioncorrection and dose-weighting, and GCTF (Zhang, 2016) was used to estimate micrograph defocus values. After triaging micrographs, particles were autopicked and 2D-classification was used to remove junk particles. The negative-stain structure of the TRAPPIII complex (Tan et al., 2013) was initially used as a reference model for 3Drefinement. After iterative rounds of 3D-classification and 3D-refinement, it was evident 
that the resulting 3D-reconstructions were quite anisotropic due to a strong orientation bias of the particles. Alternative particle-picking strategies did not provide additional orientations and therefore we decided to collect data from tilted grids.

\section{$30^{\circ}$-tilt data:}

A dataset of 430 micrographs was collected on the same microscope with the stage tilted to $30^{\circ}$. An additional 2015 micrographs were collected at the Diamond Light Source / eBIC on a Titan Krios operated at 300kV with a K2 detector and energy filter (Gatan). The image pixel size on the K2 detector was $0.824 \AA$ /pixel after binning superresolution images. Data were processed as described above for the "flat" data, with an additional step of per-particle defocus estimation using GCTF prior to 2D classification.

Each dataset was initially processed independently, producing reconstructions of $\sim 4.5 \AA$ resolution using the 0.143 FSC criterion. The datasets were then processed together, subjected to multiple 3D-classification and 3D-refinement procedures, and after CTF-Refinement and Bayesian Polishing ultimately resulted in a 3D-reconstruction with a 0.143 FSC resolution of $3.8 \AA$. However, this $3 D-$ reconstruction was still fairly anisotropic and interpretation of the distal ends of the map was further confounded by some flexibility of the complex that could not be surmounted by focused local refinements or classifications. We therefore decided to collect additional data at even higher tilt angles, as the Lyumkis group demonstrated that $40^{\circ}$ tilt was feasible for data collection (Tan et al., 2017). 


\section{$40^{\circ}$-tilt and $45^{\circ}$-tilt data:}

The Cornell CCMR facility Talos Arctica (TFS) with K3 detector and energy filter (Gatan) was used to collected 3062 micrographs on $45^{\circ}$-tilted grids and 1950 micrographs on $40^{\circ}$-tilted grids. The microscope was operated at $200 \mathrm{kV}$ at a nominal maginification of $63 \mathrm{kX}$ resulting in an image pixel size of $1.24 \AA /$ pixel. Only a small fraction of these micrographs was suitable for use, either due to extensive beaminduced motion that could not be corrected, or insufficient contrast. CryoSparc (Punjani et al., 2017) was used to triage micrographs after "Patch-CTF" micrograph estimation. CryoSparc was also used for 2D classification and initial 3D-classifications to generate a clean particle set that was then imported into RELION with the use of the csparc2star python script written by D. Asarnow (https://github.com/asarnow/pyem). RELION 3.1 was used to combine the particles imaged at $30^{\circ}, 40^{\circ}$, and $45^{\circ}$, using the scheme shown in Figure S3. Extensive 3D-classification was used to select the best set of particles. In order to deal with apparent flexibility of Trs85 relative to the rest of the complex, we employed Trs85-focused particle subtraction and 3D-classification. CTFrefinement and Bayesian polishing were also used, culminating in a final 3D reconstruction with a 0.143 FSC resolution of $3.7 \AA$.

Density modification using RESOLVE (Terwilliger et al., 2020) in Phenix (Liebschner et al., 2019) was used to facilitate interpretation of the reconstruction. The resulting map was reported by RESOLVE to have a resolution of $3.5 \AA$. In addition, focused local refinement was performed separately on Trs85, the distal region surrounding Trs33, and the central region surrounding Rab1/Ypt1 to generate additional maps to assist with model building. 
Particle orientation distributions were assessed and plotted using scripts from $A$. Leschziner (https://github.com/leschzinerlab/Relion) and D. Lyumkis et al. (https://github.com/nysbc/Anisotropy).

Model building and refinement

Available crystal structures (Kim et al., 2005; Rak et al., 2003; Cai et al., 2008) for the core subunits and Rab1/Ypt1 were docked into the map and rebuilt using Coot (Emsley et al., 2010). trRosetta (Yang et al., 2020) was used to generate models of Trs85, and portions of these models were docked or used as guides for de novo model building. Phenix was used for real-space refinement (Liebschner et al., 2019).

Restrained molecular dynamics simulations were performed using ISOLDE (Croll, 2018) within ChimeraX (Goddard et al., 2018) in order to identify and fix model errors arising due to imposition of Ramachandran restraints during refinement. The final refinement was carried out using one half-map and validation was performed using the other halfmap. The model-map 0.143 FSC values were: $3.1 \AA$ (whole-map), $3.2 \AA$ (refinement halfmap), and 3.7A (validation half-map). Model-map FSC curves are shown in Figure S2 and other validation, model, and data statistics are shown in Table 1. PyMol (Schrodinger), Chimera (Pettersen et al., 2004), and ChimeraX were used to produce images for figures.

\section{Surface analyses}

Conservation analysis was carried out using the Consurf web server utilizing custom alignments generated with Clustal Omega (Armon et al., 2001; Landau et al., 
2005; Ashkenazy et al., 2010; Celniker et al., 2013; Ashkenazy et al., 2016). The electrostatic surface potential was calculated using the APBS plugin for Pymol (Schrodinger) and the map was colored on a red $(-5 \mathrm{kT} / \mathrm{e})$ to blue $(+5 \mathrm{kT} / \mathrm{e})$ scale (Baker et al., 2001; Dolinsky et al., 2007).

\section{Sequence alignment}

The multiple sequence alignments were performed using Clustal Omega with default settings (Madeira et al., 2019) and then manually adjusted for fine-tuning of the amphipathic helix region.

Cross-linking mass spectrometry (XL-MS)

100ug of purified TRAPPIII-Rab1/Ypt1 complex (in 10mM HEPES pH 8, 150mM $\mathrm{NaCl}, 1 \mathrm{mM}$ DTT) was incubated on ice for $1 \mathrm{hr}$ with a range of disuccinimidyl sulfoxide (DSSO) concentrations spanning $0.3125 \mathrm{mM}$ to $2.5 \mathrm{mM}$. The reaction was then quenched with $10 \mathrm{mM}$ Tris $\mathrm{pH} 8$ for 15 minutes at room temperature. $10 \%$ of the sample was analyzed by SDS-PAGE (Figure S1B), and the sample cross-linked with $625 \mu \mathrm{M}$ DSSO was chosen for further analysis. To prepare the sample for mass spectrometry, the cross-linked proteins were first dried completely in a vacuum concentrator and then resuspended in $8 \mathrm{M}$ Urea, 50mM Tris $\mathrm{pH} 8$, and 5mM DTT. Urea-solubilized proteins were then incubated at $37^{\circ} \mathrm{C}$ for $30 \mathrm{~min}$ for denaturation and to reduce disulfide bridges. Reduced cysteines were blocked with $25 \mathrm{mM}$ iodoacetamide for $15 \mathrm{~min}$. Urea was then diluted to $2 \mathrm{M}$ with a solution of $50 \mathrm{mM}$ Tris $\mathrm{pH} 8$ and $150 \mathrm{mM} \mathrm{NaCl}$. Proteins were digested overnight with gentle nutation at $37^{\circ} \mathrm{C}$ using $1 \mathrm{ug}$ of Promega Trypsin GOLDß. 
The next day, the digested sample was acidified with $0.25 \%$ formic acid $+0.25 \%$ trifluoroacetic acid and desalted using a Waters Sep-Pak column. The desalted, digested sample was dried in a vacuum concentrator and reconstituted in $0.1 \%$ TFA +1 picomole/uL Angiotensin-II peptide (Sigma). 1\% of the sample was subjected to LCMS/MS/MS analysis on an Orbitrap Fusion Lumos mass spectrometer (ThermoFisher Scientific). On-line sample fractionation was performed with linear gradients of $0.1 \%$ formic acid (solvent $\mathrm{A}$ ) and $80 \%$ acetonitrile $+0.1 \%$ formic acid (solvent $\mathrm{B}$ ) at a flow rate of $300 \mathrm{nl} / \mathrm{min}$ using a home-made $125-\mu \mathrm{m} \times 25-\mathrm{cm}$ capillary column packed with $3-\mu \mathrm{m}$ C18 resin. Precursor ions were detected in the Orbitrap mass analyzer (375-1500Th, resolution 60,000 at $375 T h)$. Charge states in the $4+$ to $8+$ range were selected for MS2 analysis in the Orbitrap (resolution set at 30,000 at 375Th) with $25 \%$ CID energy. Peaks with a mass difference of 31.9721Da, indicative of a DSSO cross-link, were subjected to MS3 analysis. For MS3, ions were fragmented in the linear ion trap using higher-energy collisional dissociation (HCD) at 35\% HCD energy. The cross-link search was performed using MaXLinker software (Yugandhar et al., 2020). The structure mapping of cross-links was performed using Xlink Analyzer (Kosinski et al., 2015) implemented in UCSF Chimera (Pettersen et al., 2004).

\section{Liposome preparation}

Synthetic liposomes were prepared using a mixture of lipids (Table S4). Lipids were combined, dried by rotary evaporation, rehydrated overnight in HK buffer (20mM HEPES, pH 7.4 , and $160 \mathrm{mM} \mathrm{KOAc}$ ) at $37^{\circ} \mathrm{C}$, then extruded through $100 \mathrm{~nm}$ or $400 \mathrm{~nm}$ 
filters (Whatman) to generate homogenous liposomes. Synthetic liposomes were stored at $4^{\circ} \mathrm{C}$ until needed.

\section{Pelleting assays}

In $40 \mathrm{uL}$ reactions, loaded $\sim 1-2 \mathrm{ug}$ of protein $(300 \mathrm{nM})$ along with $500 \mu \mathrm{M}$ of $400 \mathrm{~nm}$ liposomes in HK buffer. Incubated for 15 minutes at RT. Samples were ultracentrifuged for 10 minutes at 4C, 55K rpm (TLA 100.3 rotor). The supernatant and pellet fractions were recovered and evaluated separately via SDS-PAGE. Each assay was performed at least three times. The gels shown are representative of all three experiments. The percent of pelleted protein is calculated by dividing the intensity of the pelleted bands by the total intensity of protein in both the supernatant and pelleted fractions. Then the average amount of protein that pellets in the absence of liposomes is subtracted from each sample. The quantification is based on the averages of all three experiments. All direct comparisons were performed using the same batch of liposomes to account for batch variability.

\section{5-FOA complementation assay}

Corresponding plasmids harboring mutant versions of Trs85 were transformed into a sensitized strain background (trs854::KAN, Bet3-GFP::HIS (CFY3307)), grown on -LEU plates. Several colonies were resuspended in water then serially diluted and replicated onto -LEU and 5FOA plates. Cells were grown for 48 hours at 26, 30, or $37^{\circ} \mathrm{C}$. 


\section{Microscopy}

Plasmids harboring mutant versions of Trs85 were transformed into a trs854::KANMX strain (CFY2692) so that only the plasmid copy of Trs85 would be present. For experiments assessing Rab1/Ypt1 localization, a second plasmid containing mRFPmars-Ypt1 (CFB3511) was co-transformed. Cells were grown at $30^{\circ} \mathrm{C}$ until log-phase. Cells were collected by centrifugation for 2 minutes at 12,000x g, resuspended in a small amount of media and placed on a microscope slide with coverslip. Cells were imaged at room temperature with a CSU-X spinning-disk confocal microscope system (Intelligent Imaging Innovations) on a DMI6000 microscope (Leica Microsystems) equipped with a CSU-X1 spinning-disk confocal unit (Yokogawa Electric Corporation) and a QuantEM 512SC camera (Photometrics). The objective was a 100x 1.46 NA Plan Apochromat oil immersion lens (Leica Microsystems). Additional components included a laser stack and mSAC (spherical aberration correction; Intelligent Imaging Innovations). SlideBook software was used to control the system (Intelligent Imaging Innovations) and for data analysis. All images within a figure had their minimum or maximum brightness levels adjusted equally for clarity.

\section{Co-immunoprecipitations}

Plasmids harboring Trs85 mutants were transformed into a yeast strain containing trs854::KANMX, Trs23-6xHis-3xFLAG::TRP (CFY3585). Overnight cultures were grown to saturation and then used to inoculate fresh media. Large cultures grew until log-phase and then cells were collected by centrifugation. Cell pellets were 
resuspended in $1 \mathrm{~mL}$ cold water and then transferred to an Eppendorf tube. Cell pellets were aliquoted to approximately equal weights and then the cells were collected again, flash frozen in liquid nitrogen, and stored at $-80^{\circ} \mathrm{C}$. Each colP contained roughly $250 D_{600}$ of cells per tube.

Upon removal from storage, $250 \mathrm{uL}$ of lysis buffer [50mM Tris $\mathrm{pH} 7.5,0.2 \%$ $\mathrm{NP} 40,150 \mathrm{mM} \mathrm{NaCl}, 1 \mathrm{mM}$ EDTA] and $100 \mathrm{uL}$ of $0.5 \mathrm{~mm}$ glass beads were added to each tube. Cells were immediately lysed via harsh vortexing for $2 \times 10 \mathrm{~min}$ at $4^{\circ} \mathrm{C}$, with $2 \mathrm{~min}$ rest on ice in between. Lysed cell debris was pelleted for $5 \mathrm{~min}$ at $\sim 21 \mathrm{~K} \times \mathrm{g}$ at $4{ }^{\circ} \mathrm{C}$ and the supernatant was collected. $25 \mathrm{uL}$ supernatant was used for SDS-PAGE analysis as the whole cell extract. The remaining lysate was incubated with $12.5 \mathrm{uL}$ equilibrated magnetic mNeonGreen-Trap resin (Chromotek) for a minimum of 1 hour rotating at $4^{\circ} \mathrm{C}$. After incubation, the resin was collected and washed $3 \times 1 \mathrm{~mL}$ with lysis buffer and transferred once to a new tube. Finally, 50uL of 2x SDS sample buffer was added to the resin and incubated at least $10 \mathrm{~min}$ at $95^{\circ} \mathrm{C}$. Samples were subjected to SDS-PAGE and transferred to a PVDF membrane (Millipore) for Western blot analysis probing for HA and FLAG epitopes using $1^{\circ}$ antibody = 1:500 mouse $\alpha \mathrm{HA}$ (Roche), or 1:500 mouse $\alpha$ FLAG (Roche) and $2^{\circ}$ 1:5,000 sheepamouse (Amersham).

\section{GFP-Atg8 processing assay}

Plasmids harboring Trs85 mutants were co-transformed along with GFP-Atg8 (CFB2250) into trs854::KAN (CFY2692). Overnight cultures were grown to saturation at $30^{\circ} \mathrm{C}$ and then used to inoculate fresh media. Once back in log-phase, $\sim 5-100 D_{600}$ units 
of cells were collected cells by centrifugation, $3 \mathrm{~min}$ at $\sim 3 \mathrm{k}$ rpm and processed for TCA precipitation. With the remaining culture, autophagy was induced by addition of rapamycin to a concentration of $0.2 \mathrm{uM}$. After $1 \mathrm{~h}$ of incubation at growth temperature, an additional $\sim 5-100 D_{600}$ units of cells were collected and processed for TCA precipitation. Samples were resolved by SDS-PAGE and then subjected to Western blot analysis using $1^{\circ}, 1: 1,000$ mouse $\alpha$ GFP (Roche) and $2^{\circ}, 1: 5,000$ sheep $\alpha$ mouse (Amersham). Autophagy efficiency is quantified measuring the amount of free GFP compared to the total amount of GFP-Atg8 and GFP. The blots shown are representative of all three experiments. The percent of free GFP is calculated by dividing the intensity of the free GFP band by the total intensity of both GFP-Atg8 and free GFP in the rapamycininduced lane. The quantification is based on the averages of all three experiments.

\section{Prenylated Rab1/Ypt1 preparation}

Performed as described previously (Thomas et al., 2018). Briefly, Rab/GDI substrates, the mantGDP-loaded Rab, Gdi1, His6-Mrs6, and His6-Bet2/Bet4 were mixed in a 10:10:1:1 molar ratio with a 6-fold excess of geranylgeranyl pyrophosphate (Cayman) in prenylation buffer with $20 \mu \mathrm{M}$ mantGDP and incubated at $37^{\circ} \mathrm{C}$ for $1 \mathrm{~h}$. Then, imidazole was added for a final concentration of $10 \mathrm{mM}$. The solution was incubated with a small volume of Ni-NTA resin (Qiagen) at $4^{\circ} \mathrm{C}$ for $1 \mathrm{~h}$ to remove enzymes. Stoichiometric Rab/GDI complexes were then isolated using size exclusion chromatography. 


\section{$\underline{\text { Rab1/Ypt1 nucleotide loading for GEF assays }}$}

1mg of purified Rab1/Ypt1 (either prenylated-Ypt1/GDI complex or nonprenylated Ypt1-7xHis) was incubated in the presence of 20 $\mu \mathrm{M}$ mantGDP (Sigma) and EDTA to facilitate exchange for 30 minutes at $30^{\circ} \mathrm{C}$. Afterwards, protein was buffer exchanged in $15 x$ excess buffer to remove leftover nucleotide and EDTA before being aliquoted and stored at $-80^{\circ} \mathrm{C}$.

\section{$\underline{\text { In vitro nucleotide exchange (GEF) assays }}$}

For all assays, nucleotide exchange was measured by quenched fluorescence of mantGDP (Sigma) (360nm excitation and 440nm emission). Reactions were performed inside of a fluorometer (Photon Technology International) at $30^{\circ} \mathrm{C}$ with the sequential addition of: $333 \mu \mathrm{M}$ synthetic liposomes (100nm), $200 \mu \mathrm{M}$ nonfluorescent GTP, and 250nM prenylated mantGDP:Ypt1 to HKM buffer (20mM HEPES pH 7.4, 160mM $\mathrm{KoAC}, 2 \mathrm{mM} \mathrm{MgCl} 2$ ). The mixture was then incubated at $30^{\circ} \mathrm{C}$ for $2 \mathrm{~min}$ to allow the Rab/GDI substrate time to equilibrate with the membrane. Nucleotide exchange was monitored after the addition of 20nM GEF complex. For soluble mantGDP:Ypt1-7His, identical conditions were utilized except the liposomes were replaced by HKM buffer. To determine exchange rates, traces of fluorescence versus time were fit to a one phase decay curve with an additional linear drift term using Prism (GraphPad Software). The resulting rate constants were divided by the GEF concentration to obtain the exchange rate. 
bioRxiv preprint doi: https://doi.org/10.1101/2020.10.08.332312; this version posted October 8, 2020. The copyright holder for this preprint

(which was not certified by peer review) is the author/funder, who has granted bioRxiv a license to display the preprint in perpetuity. It is made available under aCC-BY-NC-ND 4.0 International license.

\section{Software}

Structural biology software packages are maintained in our laboratory by SBGrid

(Morin et al., 2013).

Data deposition

Data will be deposited in the RCSB PDB, EMDB, and PRIDE databases. 


\section{AUTHOR CONTRIBUTIONS}

AMNJ - conceptualization and design, data acquisition/investigation (cryo-EM, XL-MS, fluorescence microscopy, cell-based assays, in vitro assays), data analysis/interpretation, writing; BPP - data acquisition (cryo-EM); KY - data acquisition (XL-MS); EJS - data acquisition (XL-MS); MBS - funding acquisition, data interpretation; $\mathrm{HY}$ - funding acquisition, data interpretation; EAM - funding acquisition, data interpretation; JCF - funding acquisition, conceptualization and design, data analysis/interpretation, writing. 


\section{ACKNOWLEDGEMENTS}

We acknowledge the MRC-LMB Electron Microscopy Facility, Diamond Light Source, and Cornell Center for Materials Research (CCMR) for access and support of electron microscopy sample preparation and data collection. We particularly thank Giuseppe Cannone, Rebecca Voorhees, Mariena Silvestry-Ramos, and Katherine Spoth for assistance with cryo-EM data collection. We also thank Bridget Carragher and Zhening Zhang of the NYSBC, and Chen Xu of the UMass Cryo-EM facility, for performing preliminary cryo-EM data collection. We thank Uche Chukwukere, Jeffrey Ho, and Kelly Rosch for aiding with preliminary cell biological experiments, Ting-Yi Wang for assisting with mass spectrometer maintenance, and Laura Thomas for generating preliminary biochemical data. We thank Daniel Asarnow, Andres Leschziner, and Dmitry Lyumkis for making scripts publicly available on GitHub. This work was supported by National Institutes of Health grants R01GM097272 and R01HD095296 to M.B.S., R01GM098621 and R35GM136258 to J.C.F., by an Alfred P. Sloan Foundation Fellowship and a National Science Foundation Graduate Research Fellowship (grant DGE-1650441) to A.M.N.J., and the UK Medical Research Council (MRC_UP_1201/10) to E.A.M. Any opinions, findings, conclusions, or recommendations expressed in this material are those of the authors and do not necessarily reflect the views of the funders. This work made use of the Cornell Center for Materials Research Shared Facilities which are supported through the NSF MRSEC program (DMR-1719875). We acknowledge Diamond for access and support of the Cryo-EM facilities at the UK national electron bio-imaging centre (eBIC), proposal EM17434, funded by the Wellcome Trust, MRC and BBSRC. 


\section{FIGURE LEGENDS}

\section{Figure 1: The Trs85 subunit anchors TRAPPIII to membranes}

A. Cartoon depicting the TRAPPIII complex subunits and the TRAPP core subunits. Trs85 (purple) is the TRAPPIII-specific subunit.

B. In vitro nucleotide exchange assay of prenylated-Rab1/Ypt1-GDI substrate in the presence of synthetic liposomes. The TRAPPIII complex (purple) is significantly more active than the TRAPP core alone (yellow).

C. Assays performed as in B, but using a non-prenylated Rab1/Ypt1 substrate and in the absence of synthetic liposomes. Exchange activity with and without the Trs85 subunit are equivalent.

D. In vitro membrane pelleting assay comparing the TRAPPIII complex versus the core subunits. While both complexes exhibit a small amount of pelleting in the absence of synthetic liposomes, in their presence only the TRAPPIII complex is strongly enriched in the membrane-bound fraction. ( $S=$ supernatant, $P=$ pellet)

E. Quantification of D. Average \% membrane binding between three assays is plotted, the error bars represent the $95 \%$ confidence intervals. The value for each replicate is depicted. The negative values arise from subtraction of the average amount of protein that pellets in the absence of liposomes.

\section{Figure 2: Cryo-EM structure of the TRAPPIII-Rab1/Ypt1 nucleotide exchange}

\section{complex}

A. Cryo-EM density map colored by each subunit. Bottom panel is rotated $90^{\circ}$ relative to the top panel. 
B. Colored as in A, the refined atomic model of the TRAPPIII complex.

C. Network map depicting all of the 108 cross-links found between the TRAPPIII complex subunits and Rab1/Ypt1. Blue lines are indicative of inter-protein crosslinks, red lines denote cross-links detected within a single protein. The relative position of the cross-link represents its location within the primary sequence.

D. The fraction of total cross-linking sites observed in each polypeptide.

E. The cross-links mapped onto the refined model. Cross-links are shown as black lines connecting black spheres.

F. A histogram depicting the length of each cross-link that was mapped to the structural model. The distance was measured between the $\alpha$-carbons of each pair of cross-linked residues. Only one of the cross-links is beyond the maximum theoretical restraint $(30 \AA)$ of the cross-linking chemical, DSSO.

\section{Figure 3: Architecture and conservation of the Trs85 subunit}

A. The Trs85 subunit colored from N- (blue) to C-terminus (red). Several features are highlighted and described in the text.

B. Surface view of Trs85 colored by ConSurf analysis. On the left is the conservation across model organisms and humans, on the right conservation was estimated based on more closely related species. The TRAPP core binding region is highly conserved. 


\section{Figure 4: The C-terminus of Trs85 binds to the TRAPP core}

A. Representative Coomassie-stained gel of size exclusion chromatography fractions after purification of recombinant wild-type TRAPPIII complex.

B. As in A, using a Trs85 mutant containing only the final 198 amino acids. NOTE: Trs85[501-698] is $\sim 25 \mathrm{kD}$ in size and now migrates at the same size as some of the core subunits.

C. As in A/B, using a Trs85 mutant with a truncation of the loop from 575-603.

$\operatorname{Trs} 85[\Delta 575-603]$ appears to dissociate from the core during chromatography, indicating a reduced affinity for the core subunits.

D. Network map showing the relative positions of cross-links between the Cterminus of Trs 85 and the core subunits Trs 31 and Trs20.

E. View of the cryo-EM structural model showing the interaction surface between Trs85, Trs31 and Trs20. Inset depicts several basic residues from Trs85 that are in close proximity to several acidic residues of Trs20.

F. Yeast complementation assays performed in a sensitized strain (BET3GFP::HIS3, trs854::KANMX) to assess the functionality of Trs85 mutants within the region shown in $\mathrm{E}$.

G. Coimmunoprecipitations of Trs23-6xHis-3xFLAG with the same Trs85 mutants.

H. Live cell fluorescence microscopy of Trs85-mNeonGreen and mRFPmars-Ypt1. Localization of Rab1/Ypt1 to punctate structures is indicative of its activation by TRAPPIII. Scale bar is 2 microns. 


\section{Figure 5: The Rab1/Ypt1 HVD points to the membrane binding surface}

A. Cryo-EM density is shown at a low threshold to highlight the almost continuous density connecting the Rab1/Ypt1 nucleotide-binding domain to its HVD. Inset is a closeup of the density corresponding to the Rab1/Ypt1 HVD region. Colored by subunit as in Figure 2, Rab1/Ypt1 is yellow. Density is depicted as it is likely oriented at the surface of organelle membranes.

B. 2D class averages showing extra density in this region in the presence of Rab1/Ypt1. Circle highlights the Rab1/Ypt1 NBD, and the arrow points to the HVD density.

C. Network diagram of cross-links observed between Trs31 and Rab1/Ypt1. Many cross-links are observed at the C-terminal region of Rab1/Ypt1. Cross-links involving residues present in the atomic model are colored black, while crosslinks between unmodeled residues are colored pink.

D. As in C, but the cross-links are mapped onto the structure. Cross-links involving residues present in the atomic model are colored black, while cross-links between unmodeled residues are colored pink.

E. Surface representation of the TRAPPIII-Rab1/Ypt1 complex colored by electrostatic potential. Top panel is oriented as in A, the bottom panel is rotated $90^{\circ}$ to visualize the most positive surface which likely lies at the surface of the membrane.

F. Quantification of in vitro TRAPPIII complex membrane pelleting assays performed with various lipid compositions. The strongest binding was observed 
with the most negatively charged liposomes. Binding is compared to wild-type on

"Golgi" liposomes using t-test with Welch's correction, * $p<0.05$, ** $p<0.01$.

\section{Figure 6: TRAPPIII uses a conserved amphipathic helix to bind the membrane}

A. Refined atomic model of the TRAPPIII complex shown as it likely interacts with membranes. The arrow points to the location of the unmodeled conserved region.

B. Sequence alignment of the unmodeled region predicted to lie on the membranebinding surface. Red helix above represents the region predicted to fold as an $\alpha$ helix in secondary structure analysis. Positively charged residues are highlighted in blue, while bulky aromatic residues are underlined.

C. Helical wheel representation of the region predicted to be an $\alpha$-helix. Positively charged residues are colored blue, polar uncharged residues are colored green, and nonpolar residues are colored yellow.

D. The wild-type Trs85 loop sequence is shown on top, with targets of mutation colored purple and mutants color coded.

E. Yeast complementation assays performed in a sensitized strain (BET3GFP::HIS3, trs854::KANMX) to assess the functionality of Trs85 mutants.

F. Coimmunoprecipitations of Trs23-6xHis-3xFLAG with the same Trs85 mutants.

G. In vitro membrane pelleting assay examining two Trs85 mutants in the context of the TRAPPIII complex. In the absence of liposomes, all three complexes pellet slightly, however in the presence of membranes, only the wild-type Trs85- 
TRAPPIII complex is strongly enriched in the pelleted fraction. ( $\mathrm{S}=$ supernatant, $\mathrm{P}=$ pellet)

H. Quantification of H. Average \% protein pelleted between three assays is plotted, the error bars represent the $95 \%$ confidence intervals. The value for each replicate is depicted. The negative values arise from subtraction of the average amount of protein that pellets in the absence of liposomes.

I. Live cell fluorescence microscopy of Trs85-mNeonGreen mutants and mRFPmars-Ypt1. Scale bar $=2 \mu \mathrm{m}$.

J. In vitro nucleotide exchange assay of prenylated-Ypt1/GDI substrate in the presence of synthetic liposomes. The wild-type Trs85-TRAPPIII complex (purple) is significantly more active than the mutants (red and black) or the core alone (yellow).

$\mathrm{K}$. Assays performed as in $\mathrm{B}$, but using a non-prenylated Rab1/Ypt1 substrate and in the absence of liposomes. Exchange activity of all complexes are equivalent.

\section{Figure 7: The membrane-binding helix is critical for autophagy}

A. GFP-Atg8 processing is monitored for either the TRAPP core binding mutations or the amphipathic helix (membrane binding) mutations. With functional Trs85, there is an accumulation of free GFP. Samples are from pre-rapamycin treatment $(-)$ and $1 \mathrm{hr}$ after addition of rapamycin (+).

B. Quantification of A. The percent of free GFP for wild-type Trs85 is compared to each mutant using t-test with Welch's correction, ${ }^{* *} p<0.01,{ }^{* * *} p<0.005$. The 
average of at least three replicates is plotted with error bars representing the 95\% confidence interval.

C. Model for TRAPPIII-mediated Rab1 activation in cells. Inactive Rab1-GDP/GDI exists in an equilibrium where Rab1-GDP samples various organellar membranes. TRAPPIII is recruited to specific membranes containing negative charges (red circles) and utilizes an amphipathic helix to bind stably to the membrane surface. Rab1 is locally activated by TRAPPIII, then recruits downstream effectors to facilitate trafficking or autophagic events.

Figure S1: Purification of the complex and cross-linking for mass spectrometry

A. Normalized UV trace from SEC to separate TRAPPIII-Rab1/Ypt1 complex from excess Rab1/Ypt1. Coomassie-stained gel corresponding to the peaks in the trace.

B. Coomassie-stained gel showing the cross-linked TRAPPIII-Rab1/Ypt1 protein sample over a range of DSSO concentrations. The $625 \mu \mathrm{M}$ sample (third lane from the right) was ultimately used for MS3 analysis and database searches.

\section{Figure S2: Cryo-EM data}

A. Example micrograph generated from a flat dataset acquisition, $1.34 \AA /$ pixel, -3.0 microns estimated average defocus.

B. Representative 2D class averages from the flat dataset generated in RELION. The edge of each box corresponds to $482 \AA$. $~ 95 \%$ of the particles are found in the 2D classes equivalent to the Ypt1-bound class shown in Figure 5B. 
C. Example micrograph generated from a $40^{\circ}$ tilt dataset acquisition, $1.24 \AA / p i x e l,-$ 1.5 microns estimated average defocus.

D. Representative 2D class averages from the combined tilt datasets generated in RELION. The edge of each box corresponds to $397 \AA$.

E. FSC curves for each of the final refined particle sets used for 3D reconstructions (see Figure S3).

F. Orientation distributions of each of the final refined particle sets used for 3D reconstructions (see Figure S3). Generated using a script written by A. Leschziner available on GitHub.

\section{Figure S3: Cryo-EM data processing flowchart}

Flow chart showing the steps taken during data processing to generate the highresolution TRAPPIII-Rab1/Ypt1 3D reconstruction. "Sphericity" is a measure of data isotropy (lower values represent more anisotropy) and was calculated using a 3DFSC script written by D. Lyumkis available on GitHub. The insets show the EM density from each of the final 3D reconstructions, surrounding the portion of Trs85 that contacts Trs20.

\section{Figure S4: Comparisons to published crystal structures}

A. Bet3 subunit from our cryo-EM reconstruction aligned with the Bet3 subunit from pdb:3CUE.

B. The second Bet3 subunit from our cryo-EM reconstruction aligned to the second Bet3 subunit from pdb:3CUE. 
C. Bet5 subunit from our cryo-EM reconstruction aligned with the Bet5 subunit from pdb:3CUE.

D. Trs23 subunit from our cryo-EM reconstruction aligned with the Trs23 subunit from pdb:3CUE.

E. Trs31 subunit from our cryo-EM reconstruction aligned with the Trs31 subunit from pdb:3CUE.

F. Trs33 subunit from our cryo-EM reconstruction aligned with the TRAPPC6 (Trs33) subunit from pdb:2C0J.

G. Trs20 subunit from our cryo-EM reconstruction aligned with the Sedlin (TRAPPC2, Trs20) subunit from pdb:1H3Q.

H. Rab1/Ypt1 subunit from our cryo-EM reconstruction aligned with the Rab1/Ypt1 subunit from pdb:3CUE. 


\section{Table S1: TRAPP complex composition}

Note: Both TRAPPII and TRAPPIII share a core set of subunits (green, middle column) and then contain several unique ones. The yeast names are shown first, with the names of the human proteins in parentheses.

\begin{tabular}{|c|c|c|}
\hline $\begin{array}{c}\text { TRAPPIII- } \\
\text { specific } \\
\text { Trs85 } \\
\text { (TRAPPC8) }\end{array}$ & $\begin{array}{c}\text { Shared Core } \\
\text { Subunits } \\
\text { Trs33 } \\
\text { (TRAPPC6A, B) }\end{array}$ & $\begin{array}{c}\text { TRAPPII- } \\
\text { specific } \\
\text { (Trs130 } \\
\text { (TRAPPC10) }\end{array}$ \\
\hline (TrSAPPC11) & $\begin{array}{c}\text { Trs1 } \\
\text { (TRAPPC5) }\end{array}$ & $\begin{array}{c}\text { Trs20 } \\
\text { (TRAPPC9) }\end{array}$ \\
\hline (TRAPPC12) & $\begin{array}{c}\text { Trs23 } \\
\text { (TRAPPC4) }\end{array}$ & Trs65 \\
\hline & $\begin{array}{c}\text { Trs20 } \\
\text { (TRAPPC2) }\end{array}$ & $\begin{array}{c}\text { Tca17 } \\
\text { (TRAPPC2L) }\end{array}$ \\
\hline & $\begin{array}{c}\text { Bet5 } \\
\text { (TRAPPC1) }\end{array}$ \\
& $\begin{array}{c}\text { Bet3 } \\
\text { (TRAPPC3, } \\
\text { C3L) }\end{array}$ \\
\hline
\end{tabular}


Table S2: Cryo-EM model validation statistics

\begin{tabular}{|c|c|}
\hline \multicolumn{2}{|l|}{ Composition (\#) } \\
\hline Chains & 10 \\
\hline Atoms & 14935 (Hydrogens: 0) \\
\hline Residues & 1836 \\
\hline Water & 0 \\
\hline Ligands & 2 palmitate molecules \\
\hline \multicolumn{2}{|l|}{ Bonds (RMSD) } \\
\hline Length $(\AA)(\#>4 \sigma)$ & $0.005(0)$ \\
\hline Angles $\left(^{\circ}\right)(\#>4 \sigma)$ & $0.964(1)$ \\
\hline MolProbity score & 2.3 \\
\hline Clash score & 17.83 \\
\hline \multicolumn{2}{|l|}{ Ramachandran plot (\%) } \\
\hline Outliers & 0.06 \\
\hline Allowed & 10.09 \\
\hline Favored & 89.85 \\
\hline \multicolumn{2}{|c|}{ Rama-Z (Ramachandran plot Z-score, RMSD) } \\
\hline whole $(\mathrm{N}=1754)$ & $2.24(0.19)$ \\
\hline helix $(N=842)$ & $0.63(0.17)$ \\
\hline sheet $(N=231)$ & $1.60(0.33)$ \\
\hline $\operatorname{loop}(\mathrm{N}=681)$ & $2.45(0.23)$ \\
\hline Rotamer outliers (\%) & 0 \\
\hline C $\beta$ outliers $(\%)$ & 0 \\
\hline CaBLAM outliers (\%) & 1.79 \\
\hline \multicolumn{2}{|l|}{ ADP (B-factors) } \\
\hline Iso/Aniso (\#) & $14935 / 0$ \\
\hline \multicolumn{2}{|l|}{$\min / \max / m e a n$} \\
\hline Protein & $87.59 / 179.40 / 123.81$ \\
\hline Ligand & $103.94 / 151.27 / 127.61$ \\
\hline Water & --- \\
\hline \multicolumn{2}{|l|}{ Resolution Estimates $(\AA)$} \\
\hline FSC half-maps $(0.143 / 0.5)$ & $3.7 / 4.2$ \\
\hline FSC model/map $(0.143 / 0.5)$ & $3.1 / 3.8$ \\
\hline \multicolumn{2}{|l|}{ Model vs. Data } \\
\hline CC (mask) & 0.82 \\
\hline CC (box) & 0.88 \\
\hline CC (peaks) & 0.76 \\
\hline CC (volume) & 0.82 \\
\hline Mean CC for ligands & 0.72 \\
\hline
\end{tabular}


Table S3: All 108 unique cross-links identified via MaxLinker database search.

\begin{tabular}{|c|c|c|c|c|c|}
\hline Peptide A & $\begin{array}{l}\text { Protein } \\
\text { A }\end{array}$ & $\begin{array}{l}\text { Protein A } \\
\text { Position }\end{array}$ & Peptide B & $\begin{array}{l}\text { Protein } \\
\text { B }\end{array}$ & $\begin{array}{l}\text { Protein B } \\
\text { Position }\end{array}$ \\
\hline AMGEEIWkNKTEK & Bet3 & 19 & IYSESLLFkR & Trs31 & 53 \\
\hline AMGEEIWKNKTEK & Bet3 & 19 & $\begin{array}{c}\text { ASAFLSQNESSSkLSNAS } \\
\text { NSPGMLANSSTATSASA } \\
\text { NER }\end{array}$ & Trs31 & 128 \\
\hline AMGEEIWkNK & Bet3 & 19 & GDDFFkEPFTNWNK & Trs23 & 108 \\
\hline NkTEK & Bet3 & 21 & kVYcLYSDYVMK & Trs23 & 181 \\
\hline AMGEEIWKNkTEK & Bet3 & 21 & GSVkNDIR & Bet5 & 64 \\
\hline $\begin{array}{c}\text { DFNkVNDHLYSMGYNIGc } \\
\mathrm{R}\end{array}$ & Bet3 & 51 & TckTAGDFETK & Trs31 & 78 \\
\hline cENLVkTSEVLSK & Bet3 & 85 & GSVkNDIR & Bet5 & 64 \\
\hline cENLVkTSEVLSK & Bet3 & 85 & SLkAMGEEIWK & Bet3 & 11 \\
\hline cENLVkTSEVLSK & Bet3 & 85 & QPTIATTDKPDRPK & Trs23 & 160 \\
\hline GDSQTEIkVK & Bet3 & 177 & $\begin{array}{c}\text { SDGYEYTVGPKQAITSEA } \\
\text { STTYIPSR }\end{array}$ & Trs31 & 29 \\
\hline VkLNR & Bet3 & 179 & LkIEEHHTVDIIR & Trs33 & 89 \\
\hline VkLNR & Bet3 & 179 & $\begin{array}{c}\text { IIQPSASDQQFPGkSDGY } \\
\text { EYTVGPK }\end{array}$ & Trs31 & 18 \\
\hline LSkGSVK & Bet5 & 60 & cENLVkTSEVLSK & Bet3 & 85 \\
\hline $\begin{array}{c}\text { SFYQEVHELYVkTLMNPF } \\
\text { YK }\end{array}$ & Trs20 & 143 & LQEKQTESLSNYITK & Trs31 & 157 \\
\hline TLMNPFYkITDPIR & Trs20 & 151 & $\begin{array}{c}\text { kDNPVYEIEFTNAENPQG } \\
\text { FPQDLK }\end{array}$ & Trs20 & 11 \\
\hline kHLSK & Trs20 & 171 & LQEKQTESLSNYITK & Trs31 & 157 \\
\hline kHLSK & Trs20 & 171 & QTESLSNYITKMR & Trs31 & 168 \\
\hline kHLSK & Trs20 & 171 & $\begin{array}{c}\text { IIQPSASDQQFPGkSDGY } \\
\text { EYTVGPK }\end{array}$ & Trs31 & 18 \\
\hline NGGGNNNkHTNNEK & Trs23 & 91 & LQEKQTESLSNYITK & Trs31 & 157 \\
\hline NGGGNNNkHTNNEK & Trs23 & 91 & AMGEEIWkNKTEK & Bet3 & 19 \\
\hline NGGGNNNkHTNNEK & Trs23 & 91 & TckTAGDFETK & Trs31 & 78 \\
\hline NGGGNNNkHTNNEK & Trs23 & 91 & IYSESLLFkR & Trs31 & 53 \\
\hline NGGGNNNkHTNNEK & Trs23 & 91 & cENLVkTSEVLSK & Bet3 & 85 \\
\hline $\begin{array}{c}\text { nGGGNNNkHTNNEK;NG } \\
\text { GGnNNkHTNNEK }\end{array}$ & Trs23 & 91 & QPTIATTDKPDRPK & Trs23 & 160 \\
\hline $\begin{array}{c}\text { NGGGNnNkHTNNEK;nG } \\
\text { GGNNNkHTNNEK }\end{array}$ & Trs23 & 91 & $\begin{array}{c}\text { LGSFKGDDFFkEPFTNW } \\
\text { NK }\end{array}$ & Trs23 & 108 \\
\hline NGGGNNNkHTNNEK & Trs23 & 91 & kMVENLQ & Trs23 & 213 \\
\hline $\begin{array}{c}\text { HTNNEkLGSFKGDDFFK } \\
\text { EPFTNWNK }\end{array}$ & Trs23 & 97 & SNLFDEkVKK & Trs23 & 210 \\
\hline $\begin{array}{c}\text { HTNNEkLGSFKGDDFFK } \\
\text { EPFTNWNK }\end{array}$ & Trs23 & 97 & $\begin{array}{c}\text { SNLFDEKVkK;SNLFDEkV } \\
\text { KK }\end{array}$ & Trs23 & 212 \\
\hline $\begin{array}{c}\text { LGSFkGDDFFKEPFTNW } \\
\text { NK }\end{array}$ & Trs23 & 102 & NGGGNNNkHTNNEK & Trs23 & 91 \\
\hline $\begin{array}{c}\text { LGSFkGDDFFKEPFTNW } \\
\text { NK }\end{array}$ & Trs23 & 102 & AMGEEIWkNK & Bet3 & 19 \\
\hline
\end{tabular}




\begin{tabular}{|c|c|c|c|c|c|}
\hline $\begin{array}{c}\text { LGSFkGDDFFKEPFTNW } \\
\text { NK }\end{array}$ & Trs23 & 102 & NkTEK & Bet3 & 21 \\
\hline $\begin{array}{c}\text { LGSFkGDDFFKEPFTNW } \\
\text { NK }\end{array}$ & Trs23 & 102 & IYSESLLFkR & Trs31 & 53 \\
\hline $\begin{array}{c}\text { GDDFFKEPFTNWNkSGL } \\
\text { R }\end{array}$ & Trs23 & 116 & cENLVkTSEVLSK & Bet3 & 85 \\
\hline $\begin{array}{c}\text { LGSFKGDDFFKEPFTNW } \\
\text { NkSGLR }\end{array}$ & Trs23 & 116 & ILkDEIPIGED & Bet3 & 185 \\
\hline QPTIATTDKPDRPK & Trs23 & 160 & $\begin{array}{c}\text { GVLSSLGYSSEEVIcLASF } \\
\text { IDRPTDRPKTAFPK }\end{array}$ & Trs33 & 251 \\
\hline $\begin{array}{c}\text { SNLFDEkVKK;SNLFDEK } \\
\text { VkK }\end{array}$ & Trs23 & 210 & nGGGnNNkHTNNEK & Trs23 & 91 \\
\hline SNLFDEkVK & Trs23 & 210 & kVYcLYSDYVMK & Trs23 & 181 \\
\hline $\begin{array}{c}\text { SNLFDEKVkK;SNLFDEkV } \\
\text { KK }\end{array}$ & Trs23 & 212 & $\begin{array}{c}\text { nGGGNNNkHTNNEK;NG } \\
\text { GGNNNkHTnNEK }\end{array}$ & Trs23 & 91 \\
\hline $\begin{array}{c}\text { SNLFDEKVkK;SNLFDEkV } \\
\text { KK }\end{array}$ & Trs23 & 212 & kVYcLYSDYVMK & Trs23 & 181 \\
\hline $\begin{array}{c}\text { IIQPSASDQQFPGkSDGY } \\
\text { EYTVGPK }\end{array}$ & Trs31 & 18 & TSEVLSkcAFK & Bet3 & 92 \\
\hline $\begin{array}{c}\text { IIQPSASDQQFPGkSDGY } \\
\text { EYTVGPK }\end{array}$ & Trs31 & 18 & cENLVkTSEVLSK & Bet3 & 85 \\
\hline $\begin{array}{c}\text { IIQPSASDQQFPGkSDGY } \\
\text { EYTVGPK }\end{array}$ & $\operatorname{Trs} 31$ & 18 & LkNQLNLK & Trs85 & 670 \\
\hline $\begin{array}{c}\text { IIQPSASDQQFPGkSDGY } \\
\text { EYTVGPK }\end{array}$ & Trs31 & 18 & GDSQTEIkVK & Bet3 & 177 \\
\hline $\begin{array}{c}\text { IIQPSASDQQFPGkSDGY } \\
\text { EYTVGPK }\end{array}$ & Trs31 & 18 & TLMNPFYkITDPIR & Trs20 & 151 \\
\hline $\begin{array}{c}\text { IIQPSASDQQFPGkSDGY } \\
\text { EYTVGPK }\end{array}$ & Trs31 & 18 & SLKAMGEEIWK & Bet3 & 11 \\
\hline $\begin{array}{c}\text { IIQPSASDQQFPGkSDGY } \\
\text { EYTVGPK }\end{array}$ & Trs31 & 18 & $\begin{array}{c}\text { ASAFLSQNESSSkLSNAS } \\
\text { NSPGMLANSSTATSASA } \\
\text { NER }\end{array}$ & Trs31 & 128 \\
\hline $\begin{array}{c}\text { IIQPSASDQQFPGkSDGY } \\
\text { EYTVGPK }\end{array}$ & Trs31 & 18 & QTESLSNYITKMR & Trs31 & 168 \\
\hline TckTAGDFETK & Trs31 & 78 & cENLVkTSEVLSK & Bet3 & 85 \\
\hline TckTAGDFETK & Trs31 & 78 & TVELDGkTVK & Ypt1 & 55 \\
\hline TckTAGDFETK & Trs31 & 78 & $\begin{array}{c}\text { ESMSQQNLNETTQkKED } \\
\mathrm{K}\end{array}$ & Ypt1 & 184 \\
\hline TckTAGDFETK & $\operatorname{Trs} 31$ & 78 & QIkESMSQQNLNETTQK & Ypt1 & 170 \\
\hline TckTAGDFETK & Trs31 & 78 & EDkGNVNLK & Ypt1 & 188 \\
\hline $\begin{array}{c}\text { ASAFLSQNESSSkLSNAS } \\
\text { NSPGMLANSSTATSASA } \\
\text { NER }\end{array}$ & Trs31 & 128 & SLKAMGEEIWK & Bet3 & 11 \\
\hline $\begin{array}{c}\text { ASAFLSQNESSSkLSNAS } \\
\text { NSPGMLANSSTATSASA } \\
\text { NER }\end{array}$ & Trs31 & 128 & TSEVLSkcAFK & Bet3 & 92 \\
\hline $\begin{array}{c}\text { ASAFLSQNESSSkLSNAS } \\
\text { NSPGMLANSSTATSASA } \\
\text { NER }\end{array}$ & Trs31 & 128 & IYSESLLFkR & Trs31 & 53 \\
\hline LQEkQTESLSNYITK & Trs31 & 157 & IYSESLLFkR & Trs31 & 53 \\
\hline LQEkQTESLSNYITK & Trs31 & 157 & $\begin{array}{c}\text { ASAFLSQNESSSkLSNAS } \\
\text { NSPGmLANSSTATSASA } \\
\text { NER }\end{array}$ & Trs31 & 128 \\
\hline
\end{tabular}




\begin{tabular}{|c|c|c|c|c|c|}
\hline LQEKQTESLSNYITK & Trs31 & 157 & $\begin{array}{c}\text { SDGYEYTVGPKQAITSEA } \\
\text { STTYIPSR }\end{array}$ & Trs31 & 29 \\
\hline LQEkQTESLSNYITK & Trs31 & 157 & $\begin{array}{c}\text { IIQPSASDQQFPGkSDGY } \\
\text { EYTVGPK }\end{array}$ & Trs31 & 18 \\
\hline QTESLSNYITKMR & Trs31 & 168 & $\begin{array}{c}\text { ASAFLSQNESSSkLSNAS } \\
\text { NSPGmLANSSTATSASA } \\
\text { NER }\end{array}$ & Trs31 & 128 \\
\hline QTESLSNYITKMR & Trs31 & 168 & $\begin{array}{c}\text { SDGYEYTVGPkQAITSEA } \\
\text { STTYIPSR }\end{array}$ & Trs31 & 29 \\
\hline $\begin{array}{c}\text { QISGDVISSDSNVTSENG } \\
\text { NINNMIkR }\end{array}$ & $\operatorname{Trs} 33$ & 86 & VkLNR & Bet3 & 179 \\
\hline LkIEEHHTVDIIR & Trs33 & 89 & ILkDEIPIGED & Bet3 & 185 \\
\hline FKEMDLLLIMkFIcR & Trs33 & 158 & QIDNLkTNHR & Trs33 & 177 \\
\hline QIDNLkTNHR & Trs33 & 177 & $\begin{array}{l}\text { GVLSSLGYSSEEVICLASF } \\
\text { IDRPTDRPkTAFPK }\end{array}$ & Trs33 & 251 \\
\hline $\begin{array}{l}\text { FFGGcVSDRDQANEAkV } \\
\text { GQHEHEVcDASDSTDSIP } \\
\mathrm{K}\end{array}$ & Trs85 & 81 & NkNLEVPNLSK & Trs85 & 103 \\
\hline $\begin{array}{c}\text { VGQHEHEVcDASDSTDSI } \\
\text { PkNK }\end{array}$ & Trs85 & 101 & NLEVPNLSkK & Trs85 & 112 \\
\hline cQALAkK & Trs85 & 278 & NkNLEVPNLSK & Trs85 & 103 \\
\hline cQALAkK & Trs85 & 278 & NLEVPNLSkK & Trs85 & 112 \\
\hline kFMNK & Trs85 & 382 & KSLFNGAkFFK & Trs85 & 378 \\
\hline kFMNK & Trs85 & 382 & kSLFNGAK & Trs85 & 371 \\
\hline GKGkESNSLDVTEPVR & Trs85 & 502 & LIAAkK & Trs85 & 631 \\
\hline VGAMkK & Trs85 & 586 & NLEVPNLSkK & Trs85 & 112 \\
\hline DAkDTEDLRGEHK & Trs85 & 590 & VGAmkK & Trs85 & 586 \\
\hline kWAEQK & Trs85 & 632 & GKGkESNSLDVTEPVR & Trs85 & 502 \\
\hline WAEQkQWR & Trs85 & 637 & $\begin{array}{l}\text { NQLNLKDVDSAPRPSEk } \\
\text { NLTR }\end{array}$ & Trs85 & 687 \\
\hline WAEQkQWR & Trs85 & 637 & LIAAkK & Trs85 & 631 \\
\hline $\begin{array}{c}\text { DIESTYSEIkFLHGnGLILS } \\
\mathrm{K}\end{array}$ & Trs85 & 657 & LkNQLNLK & Trs85 & 670 \\
\hline $\begin{array}{c}\text { DIESTYSEIkFLHGNGLIL } \\
\text { SK }\end{array}$ & Trs85 & 657 & DAkDTEDLRGEHK & Trs85 & 590 \\
\hline $\begin{array}{c}\text { DIESTYSEIkFLHGNGLIL } \\
\text { SK }\end{array}$ & Trs85 & 657 & TLMNPFYkITDPIR & Trs20 & 151 \\
\hline $\begin{array}{c}\text { DIESTYSEIkFLHGNGLIL } \\
\text { SK }\end{array}$ & Trs85 & 657 & $\begin{array}{c}\text { kDNPVYEIEFTNAENPQG } \\
\text { FPQDLK }\end{array}$ & Trs20 & 11 \\
\hline $\begin{array}{c}\text { DIESTYSEIkFLHGNGLIL } \\
\text { SK }\end{array}$ & Trs85 & 657 & $\begin{array}{c}\text { IIQPSASDQQFPGkSDGY } \\
\text { EYTVGPK }\end{array}$ & Trs31 & 18 \\
\hline FLHGNGLILSkLK & Trs85 & 668 & NQLNLkDVDSAPRPSEK & Trs85 & 676 \\
\hline FLHGnGLILSkLK & Trs85 & 668 & LIAAkK & Trs85 & 631 \\
\hline FLHGNGLILSkLK & Trs85 & 668 & WAEQkQWR & Trs85 & 637 \\
\hline LkNQLNLK & Trs85 & 670 & QVSWcLkDIESTYSEIK & Trs85 & 647 \\
\hline LkNQLNLK & Trs85 & 670 & DVDSAPRPSEkNLTR & Trs85 & 687 \\
\hline NQLNLKDVDSAPRPSEK & Trs85 & 676 & WAEQkQWR & Trs85 & 637 \\
\hline NQLNLkDVDSAPRPSEK & Trs85 & 676 & $\begin{array}{c}\text { DIESTYSEIkFLHGNGLILS } \\
\mathrm{K}\end{array}$ & Trs85 & 657 \\
\hline NQLNLkDVDSAPRPSEK & Trs85 & 676 & LIAAkK & Trs85 & 631 \\
\hline NQLNLKDVDSAPRPSEK & Trs85 & 676 & QVSWcLkDIESTYSEIK & Trs85 & 647 \\
\hline DVDSAPRPSEkNLTR & Trs85 & 687 & FLHGNGLILSkLK & Trs85 & 668 \\
\hline
\end{tabular}




\begin{tabular}{|c|c|c|c|c|c|}
\hline DVDSAPRPSEkNLTR & Trs85 & 687 & $\begin{array}{c}\text { DIESTYSEIkFLHGnGLILS } \\
\mathrm{K}\end{array}$ & Trs85 & 657 \\
\hline $\begin{array}{c}\text { NQLNLKDVDSAPRPSEk } \\
\text { NLTR }\end{array}$ & Trs85 & 687 & LIAAkK & Trs85 & 631 \\
\hline DVDSAPRPSEkNLTR & Trs85 & 687 & QVSWcLkDIESTYSEIK & Trs85 & 647 \\
\hline DVDSAPRPSEkNLTR & Trs85 & 687 & $\begin{array}{c}\text { VGQHEHEVcDASDSTDSI } \\
\text { PkNK }\end{array}$ & Trs85 & 101 \\
\hline DVDSAPRPSEkNLTR & Trs85 & 687 & $\begin{array}{c}\text { IIQPSASDQQFPGkSDGY } \\
\text { EYTVGPK }\end{array}$ & Trs31 & 18 \\
\hline DVDSAPRPSEkNLTR & Trs85 & 687 & kWAEQK & Trs85 & 632 \\
\hline IkTVELDGK & Ypt1 & 48 & LLLIGNSGVGkScLLLR & Ypt1 & 21 \\
\hline TVELDGkTVK & Ypt1 & 55 & QIkESmSQQNLNETTQK & Ypt1 & 170 \\
\hline LLVGNkcDLK & Ypt1 & 122 & LLLIGNSGVGkScLLLR & Ypt1 & 21 \\
\hline cDLkDKR & Ypt1 & 126 & LLLIGNSGVGkScLLLR & Ypt1 & 21 \\
\hline cDLKDkR;cDLkDKR & Ypt1 & 128 & LLLIGNSGVGkScLLLR & Ypt1 & 21 \\
\hline ESMSQQNLNETTQkK & Ypt1 & 184 & EDkGNVNLK & Ypt1 & 188 \\
\hline ESMSQQNLNETTQkK & Ypt1 & 184 & TVELDGkTVK & Ypt1 & 55 \\
\hline kEDKGNVNLK & Ypt1 & 185 & TckTAGDFETK & Trs31 & 78 \\
\hline kEDKGNVNLK & Ypt1 & 185 & TVELDGkTVK & Ypt1 & 55 \\
\hline KEDkGNVNLK & Ypt1 & 188 & VkLNR & Bet3 & 179 \\
\hline
\end{tabular}


Table S4: Composition of synthetic liposomes used in this study

\begin{tabular}{|c|c|c|c|c|c|c|}
\hline & Golgi & $\begin{array}{c}\text { Golgi no } \\
\text { cholesterol }\end{array}$ & Anionic & $\begin{array}{c}\text { No } \\
\mathbf{P S} / \mathbf{P A}\end{array}$ & No PI & $\mathbf{1 0 \%} \mathbf{P I}_{\mathbf{4}} \mathbf{P}$ \\
\hline $\mathbf{P C}$ (mol \%) & 30 & 30 & 51 & 36 & 60 & 58 \\
\hline $\mathbf{P E}$ & 10 & 10 & & 10 & 10 & 10 \\
\hline $\mathbf{P S}$ & 3 & 3 & 3 & & 3 & \\
\hline $\mathbf{P A}$ & 3 & 3 & 3 & & 3 & \\
\hline $\mathbf{P I}$ & 29 & 29 & 29 & 29 & & \\
\hline $\mathbf{P I}_{\mathbf{4}} \mathbf{P}$ & 1 & 1 & 1 & 1 & & 10 \\
\hline $\mathbf{P I}_{\mathbf{4}, 5} \mathbf{P}_{\mathbf{2}}$ & & & 0.8 & & & \\
\hline $\mathbf{C D P}-\mathbf{D A G}$ & 2 & 2 & 2 & 2 & 2 & \\
\hline PO-DAG & 4 & 4 & & 4 & 4 & 4 \\
\hline DO-DAG & 2 & 2 & & 2 & 2 & 2 \\
\hline Ceramide & 5 & 5 & & 5 & 5 & 5 \\
\hline Cholesterol & 10 & & 10 & 10 & 10 & 10 \\
\hline
\end{tabular}

Note: All lipids are from Avanti Polar Lipids. $\mathrm{PC}=$ phosphatidylcholine, $\mathrm{PE}=$ phosphatidylethanolamine, $\mathrm{PS}=$ phosphatidylserine, $\mathrm{PA}=$ phosphatidic acid, $\mathrm{PI}=$ phosphatidylinositol, $\mathrm{Pl}_{4} \mathrm{P}=$ phosphatidylinositol 4-phosphate, $\mathrm{Pl}_{4,5} \mathrm{P}_{2}=$ phosphatidylinositol 4,5-ibsphosphate, CDP-DAG = cytidine diphosphate diacylglycerol, PO-DAG = palmitoyl-oleoyl diacylglycerol, DO-DAG = dioleoyl diacylglcerol 
Table S5: Plasmid list

\begin{tabular}{|c|c|c|c|}
\hline Name & Description & Vector & Source \\
\hline pLT21 & Trs33, Trs31, Trs23, Trs20, Bet3, Bet5 & pColaDuet-1 & $\begin{array}{l}\text { (Thomas and } \\
\text { Fromme, } \\
\text { 2016) }\end{array}$ \\
\hline pLT35 & 6xHisTEV-Mrs6 & pET28 & $\begin{array}{l}\text { (Thomas and } \\
\text { Fromme, } \\
2016)\end{array}$ \\
\hline pLT40 & GST-Gdi1 with cleavable tag & pGEX-6P & $\begin{array}{c}\text { (Thomas and } \\
\text { Fromme, } \\
\text { 2016) }\end{array}$ \\
\hline pLT41 & 6xHisTEV-Bet2, Bet4 & pCDF-Duet-1 & $\begin{array}{c}\text { (Thomas and } \\
\text { Fromme, } \\
2016)\end{array}$ \\
\hline pLT50 & GST-Ypt1 with cleavable tag & pGEX-6P & $\begin{array}{c}\text { (Thomas and } \\
\text { Fromme, } \\
2016)\end{array}$ \\
\hline pRS415 & Yeast centromeric vector with LEU2 marker & pRS415 & $\begin{array}{l}\text { (Sikorski and } \\
\text { Hieter, 1989) }\end{array}$ \\
\hline Ypt1-7His & GST-Ypt1-7xHis cleavable N-terminal tag & pGEX-6P & $\begin{array}{c}\text { (McDonold and } \\
\text { Fromme, } \\
2014)\end{array}$ \\
\hline CFB2250 & GFP-Atg8 & pRS416 & $\begin{array}{l}\text { (Guan et al., } \\
\text { 2001) }\end{array}$ \\
\hline CFB2730 & 6xHis-Trs85[501-698] & pETDuet-1 & This study \\
\hline CFB2868 & 6xHis-Trs85, Trs33 & pETDuet-1 & This study \\
\hline CFB2991 & Trs85-mNeonGreen-3xHA & pRS415 & This study \\
\hline CFB3125 & $\begin{array}{l}\text { Trs85[R618E, R619E, R620E, K621E]- } \\
\text { mNeonGreen-3xHA }\end{array}$ & pRS415 & This study \\
\hline CFB3200 & $\begin{array}{c}\text { Trs85[R618A, R619A, R620A, K621A]- } \\
\text { mNeonGreen-3xHA }\end{array}$ & pRS415 & This study \\
\hline CFB3222 & Trs85[ $\Delta 507-603]-m N e o n G r e e n-3 x H A$ & pRS415 & This study \\
\hline CFB3223 & $6 x H i s-\operatorname{Trs} 85[\Delta 507-603], \operatorname{Trs} 33$ & pETDuet-1 & This study \\
\hline CFB3511 & mRFPmars-Ypt1 in pRS416 & pRS416 & This study \\
\hline CFB3710 & Trs85[501-698]-mNeonGreen-3xHA & pRS415 & This study \\
\hline CFB4191 & $\begin{array}{c}\text { Trs85[K378A, K381A, K382A, K386A]- } \\
\text { mNeonGreen-3xHA }\end{array}$ & pRS415 & This study \\
\hline CFB4192 & $\begin{array}{c}\text { Trs85[K378A, F379A, F380A, K381A, K382A, } \\
\text { F383A, K386A]-mNeonGreen-3xHA }\end{array}$ & pRS415 & This study \\
\hline CFB4193 & $\begin{array}{c}\text { Trs85[K378E, K381E, K382E, K386E]- } \\
\text { mNeonGreen-3xHA }\end{array}$ & pRS415 & This study \\
\hline CFB4194 & Trs85[R620A]-mNeonGreen-3xHA & pRS415 & This study \\
\hline CFB4195 & Trs85[R620E]-mNeonGreen-3xHA & pRS415 & This study \\
\hline CFB4227 & $\begin{array}{l}\text { 6xHis-Trs85[K378A, F379A, F380A, K381A, } \\
\text { K382A, F383A, K386A], Trs33 }\end{array}$ & pETDuet-1 & This study \\
\hline CFB4229 & $\begin{array}{l}\text { 6xHis-Trs85[K378E, K381E, K382E, K386E], } \\
\text { Trs33 }\end{array}$ & pETDuet-1 & This study \\
\hline
\end{tabular}


Table S6: Strain list

\begin{tabular}{|c|c|c|}
\hline Name & Description & Source \\
\hline SEY 6210 & $\begin{array}{l}\text { MATa ura3-52 his3- } \Delta 200 \text { leu2-3,112 lys2-801 } \\
\text { trp1- } \Delta 901 \text { suc2- } \Delta 9\end{array}$ & $\begin{array}{l}\text { (Robinson et al., } \\
\text { 1988) }\end{array}$ \\
\hline SEY 6210.1 & $\begin{array}{c}\text { MATa ura3-52 his3- } \Delta 200 \text { leu2-3,112 lys2-801 } \\
\text { trp1- } \Delta 901 \text { suc2- } \Delta 9\end{array}$ & $\begin{array}{l}\text { (Robinson et al., } \\
\text { 1988) }\end{array}$ \\
\hline CFY2449 & SEY6210.1 Trs85-mNeonGreen::HIS3 & $\begin{array}{l}\text { (Thomas et al., } \\
\text { 2018) }\end{array}$ \\
\hline CFY2692 & SEY6210 trs85 $\Delta:: K a n M X$ & $\begin{array}{l}\text { (Thomas et al., } \\
\text { 2018) }\end{array}$ \\
\hline CFY3585 & $\begin{array}{c}\text { SEY } 6210 \text { Trs23-6xHis-3xFLAG::TRP, } \\
\text { trs85 }:: \text { KanMX }\end{array}$ & This study \\
\hline CFY3307 & $\begin{array}{c}\text { SEY6210.1 Bet3-GFP::HIS3, trs85 } 6: \text { KanMX, } \\
\text { Trs85-mNeonGreen-3xHA:URA }\end{array}$ & This study \\
\hline
\end{tabular}




\section{REFERENCES}

Antonny, B., Beraud-Dufour, S., Chardin, P., and Chabre, M. (1997). N-Terminal Hydrophobic Residues of the G-Protein ADP-Ribosylation Factor-1 Insert into Membrane Phospholipids upon GDP to GTP Exchange ${ }^{\dagger}$. Biochemistry $36,4675-4684$.

Armon, A., Graur, D., and Ben-Tal, N. (2001). ConSurf: an algorithmic tool for the identification of functional regions in proteins by surface mapping of phylogenetic information. Journal of Molecular Biology 307, 447-463.

Ashkenazy, H., Erez, E., Martz, E., Pupko, T., and Ben-Tal, N. (2010). ConSurf 2010:

calculating evolutionary conservation in sequence and structure of proteins and nucleic acids. Nucleic Acids Res 38, W529-W533.

Ashkenazy, H., Abadi, S., Martz, E., Chay, O., Mayrose, I., Pupko, T., and Ben-Tal, N. (2016). ConSurf 2016: an improved methodology to estimate and visualize evolutionary conservation in macromolecules. Nucleic Acids Res 44, W344-W350.

Baker, N.A., Sept, D., Joseph, S., Holst, M.J., and McCammon, J.A. (2001). Electrostatics of nanosystems: Application to microtubules and the ribosome. PNAS 98, 10037-10041.

Bassik, M.C., Kampmann, M., Lebbink, R.J., Wang, S., Hein, M.Y., Poser, I., Weibezahn, J., Horlbeck, M.A., Chen, S., Mann, M., et al. (2013). A Systematic Mammalian Genetic Interaction Map Reveals Pathways Underlying Ricin Susceptibility. Cell 152, 909-922.

Brunet, S., Shahrzad, N., Saint-Dic, D., Dutczak, H., and Sacher, M. (2013). A trs20 Mutation That Mimics an SEDT-Causing Mutation Blocks Selective and Non-Selective Autophagy: A Model for TRAPP III Organization. Traffic 14, 1091-1104.

Cai, H., Yu, S., Menon, S., Cai, Y., Lazarova, D., Fu, C., Reinisch, K., Hay, J.C., and FerroNovick, S. (2007). TRAPPI tethers COPII vesicles by binding the coat subunit Sec23. Nature 445, 941-944.

Cai, Y., Chin, H.F., Lazarova, D., Menon, S., Fu, C., Cai, H., Sclafani, A., Rodgers, D.W., De La Cruz, E.M., Ferro-Novick, S., et al. (2008). The Structural Basis for Activation of the Rab Ypt1p by the TRAPP Membrane-Tethering Complexes. Cell 133, 1202-1213.

Cao, X., Ballew, N., and Barlowe, C. (1998). Initial docking of ER-derived vesicles requires Usolp and Yptlp but is independent of SNARE proteins. EMBO J 17, 2156-2165.

Celniker, G., Nimrod, G., Ashkenazy, H., Glaser, F., Martz, E., Mayrose, I., Pupko, T., and BenTal, N. (2013). ConSurf: Using Evolutionary Data to Raise Testable Hypotheses about Protein Function. Israel Journal of Chemistry 53, 199-206.

Chavrier, P., Gorvel, J.-P., Stelzer, E., Simons, K., Gruenberg, J., and Zerial, M. (1991). Hypervariable $\mathrm{C}$-termmal domain of rab proteins acts as a targeting signal. Nature 353, 769-772. 
Croll, T.I. (2018). ISOLDE: a physically realistic environment for model building into lowresolution electron-density maps. Acta Cryst D 74, 519-530.

Dolinsky, T.J., Czodrowski, P., Li, H., Nielsen, J.E., Jensen, J.H., Klebe, G., and Baker, N.A. (2007). PDB2PQR: expanding and upgrading automated preparation of biomolecular structures for molecular simulations. Nucleic Acids Res 35, W522-W525.

Dong, G., Medkova, M., Novick, P., and Reinisch, K.M. (2007). A Catalytic Coiled Coil: Structural Insights into the Activation of the Rab GTPase Sec4p by Sec2p. Molecular Cell 25, $455-462$.

Drin, G., and Antonny, B. (2010). Amphipathic helices and membrane curvature. FEBS Letters 584, 1840-1847.

Dunn, B., Stearns, T., and Botstein, D. (1993). Specificity domains distinguish the Ras-related GTPases Ypt1 and Sec4. Nature 362, 563-565.

Emsley, P., Lohkamp, B., Scott, W.G., and Cowtan, K. (2010). Features and development of Coot. Acta Crystallogr D Biol Crystallogr 66, 486-501.

Goddard, T.D., Huang, C.C., Meng, E.C., Pettersen, E.F., Couch, G.S., Morris, J.H., and Ferrin, T.E. (2018). UCSF ChimeraX: Meeting modern challenges in visualization and analysis. Protein Sci 27, 14-25.

Goody, R.S., Rak, A., and Alexandrov, K. (2005). The structural and mechanistic basis for recycling of Rab proteins between membrane compartments. CMLS, Cell. Mol. Life Sci. 62, $1657-1670$.

Guan, J., Stromhaug, P.E., George, M.D., Habibzadegah-Tari, P., Bevan, A., Dunn, W.A., and Klionsky, D.J. (2001). Cvt18/Gsa12 Is Required for Cytoplasm-to-Vacuole Transport, Pexophagy, and Autophagy in Saccharomyces cerevisiae andPichia pastoris. MBoC 12, 38213838.

Huang, M., Weissman, J.T., Béraud-Dufour, S., Luan, P., Wang, C., Chen, W., Aridor, M., Wilson, I.A., and Balch, W.E. (2001). Crystal structure of Sar1-GDP at $1.7 \AA$ resolution and the role of the NH2 terminus in ER export. J Cell Biol 155, 937-948.

Hutagalung, A., and Novick, P. (2011). Role of Rab GTPases in Membrane Traffic and Cell Physiology. Physiol Rev 91, 119-149.

Imai, K., Hao, F., Fujita, N., Tsuji, Y., Oe, Y., Araki, Y., Hamasaki, M., Noda, T., and Yoshimori, T. (2016). Atg9A trafficking through the recycling endosomes is required for autophagosome formation. J Cell Sci 129, 3781-3791.

Jang, S.B., Kim, Y.-G., Cho, Y.-S., Suh, P.-G., Kim, K.-H., and Oh, B.-H. (2002). Crystal Structure of SEDL and Its Implications for a Genetic Disease Spondyloepiphyseal Dysplasia Tarda. J. Biol. Chem. 277, 49863-49869. 
Jedd, G., Richardson, C.J., Litt, R.J., and Segev, N. (1995). The Ypt1 GTPase is essential for the first two steps of the yeast secretory pathway. J Cell Biol 131, 583-590.

Jones, S., Newman, C., Liu, F., and Segev, N. (2000). The TRAPP Complex Is a Nucleotide Exchanger for Ypt1 and Ypt31/32. Mol Biol Cell 11, 4403-4411.

Kahn, R.A., Randazzo, P., WeissII, O., Rulka, C., Clark, J., Amherdt, M., Roller, P., and Orci, L. (1992). The Amino Terminus of ADP-ribosylation Factor (ARF) Is a Critical Determinant of ARF Activities and Is a Potent andSpecific Inhibitor of Protein Transport. Journal of Biological Chemistry 267, 8 .

Kakuta, S., Yamamoto, H., Negishi, L., Kondo-Kakuta, C., Hayashi, N., and Ohsumi, Y. (2012). Atg9 Vesicles Recruit Vesicle-tethering Proteins Trs85 and Ypt1 to the Autophagosome Formation Site. J Biol Chem 287, 44261-44269.

Kim, M.-S., Yi, M.-J., Lee, K.-H., Wagner, J., Munger, C., Kim, Y.-G., Whiteway, M., Cygler, M., Oh, B.-H., and Sacher, M. (2005). Biochemical and Crystallographic Studies Reveal a Specific Interaction Between TRAPP Subunits Trs33p and Bet3p. Traffic 6, 1183-1195.

Kim, Y.-G., Raunser, S., Munger, C., Wagner, J., Song, Y.-L., Cygler, M., Walz, T., Oh, B.-H., and Sacher, M. (2006). The Architecture of the Multisubunit TRAPP I Complex Suggests a Model for Vesicle Tethering. Cell 127, 817-830.

Kimanius, D., Forsberg, B.O., Scheres, S.H., and Lindahl, E. (2016). Accelerated cryo-EM structure determination with parallelisation using GPUs in RELION-2. ELife 5, e18722.

Klionsky, D.J., Ait-Si-Ali, S., Akematsu, T., Akporiaye, E.T., Albaiceta, G.M., Albanese, C., Albani, D., Albert, M.L., Aldudo, J., Algül, H., et al. (2016). Guidelines for the use and interpretation of assays for monitoring autophagy (3rd edition). Autophagy 12, 1-222.

Kosinski, J., von Appen, A., Ori, A., Karius, K., Müller, C.W., and Beck, M. (2015). Xlink Analyzer: Software for analysis and visualization of cross-linking data in the context of threedimensional structures. Journal of Structural Biology 189, 177-183.

Koumandou, V.L., Dacks, J.B., Coulson, R.M., and Field, M.C. (2007). Control systems for membrane fusion in the ancestral eukaryote; evolution of tethering complexes and SM proteins. BMC Evolutionary Biology 7, 29.

Kümmel, D., Heinemann, U., and Veit, M. (2006). Unique self-palmitoylation activity of the transport protein particle component Bet3: A mechanism required for protein stability. PNAS $103,12701-12706$.

Lamb, C.A., Nühlen, S., Judith, D., Frith, D., Snijders, A.P., Behrends, C., and Tooze, S.A. (2016). TBC1D14 regulates autophagy via the TRAPP complex and ATG9 traffic. EMBO J 35, 281-301. 
Landau, M., Mayrose, I., Rosenberg, Y., Glaser, F., Martz, E., Pupko, T., and Ben-Tal, N. (2005). ConSurf 2005: the projection of evolutionary conservation scores of residues on protein structures. Nucleic Acids Res 33, W299-W302.

Lee, M.C.S., Orci, L., Hamamoto, S., Futai, E., Ravazzola, M., and Schekman, R. (2005). Sar1p N-Terminal Helix Initiates Membrane Curvature and Completes the Fission of a COPII Vesicle. Cell 122, 605-617.

Li, F., Yi, L., Zhao, L., Itzen, A., Goody, R.S., and Wu, Y.-W. (2014). The role of the hypervariable C-terminal domain in Rab GTPases membrane targeting. PNAS 111, 2572-2577.

Liebschner, D., Afonine, P.V., Baker, M.L., Bunkóczi, G., Chen, V.B., Croll, T.I., Hintze, B., Hung, L.-W., Jain, S., McCoy, A.J., et al. (2019). Macromolecular structure determination using X-rays, neutrons and electrons: recent developments in Phenix. Acta Cryst D 75, 861-877.

Lipatova, Z., Belogortseva, N., Zhang, X.Q., Kim, J., Taussig, D., and Segev, N. (2012). Regulation of selective autophagy onset by a Ypt/Rab GTPase module. PNAS 109, 6981-6986.

Lord, C., Bhandari, D., Menon, S., Ghassemian, M., Nycz, D., Hay, J., Ghosh, P., and FerroNovick, S. (2011). Sequential interactions with Sec23 control the direction of vesicle traffic. Nature 473, 181-186.

Lynch-Day, M.A., Bhandari, D., Menon, S., Huang, J., Cai, H., Bartholomew, C.R., Brumell, J.H., Ferro-Novick, S., and Klionsky, D.J. (2010). Trs85 directs a Ypt1 GEF, TRAPPIII, to the phagophore to promote autophagy. PNAS 107, 7811-7816.

Madeira, F., Park, Y. mi, Lee, J., Buso, N., Gur, T., Madhusoodanan, N., Basutkar, P., Tivey, A.R.N., Potter, S.C., Finn, R.D., et al. (2019). The EMBL-EBI search and sequence analysis tools APIs in 2019. Nucleic Acids Res 47, W636-W641.

McDonold, C.M., and Fromme, J.C. (2014). Four GTPases Differentially Regulate the Sec7 ArfGEF to Direct Traffic at the trans-Golgi Network. Dev Cell 30, 759-767.

Meiling-Wesse, K., Epple, U.D., Krick, R., Barth, H., Appelles, A., Voss, C., Eskelinen, E.-L., and Thumm, M. (2005). Trs85 (Gsg1), a Component of the TRAPP Complexes, Is Required for the Organization of the Preautophagosomal Structure during Selective Autophagy via the Cvt Pathway. J. Biol. Chem. 280, 33669-33678.

Morin, A., Eisenbraun, B., Key, J., Sanschagrin, P.C., Timony, M.A., Ottaviano, M., and Sliz, P. (2013). Cutting Edge: Collaboration gets the most out of software (eLife Sciences Publications Limited).

Morozova, N., Liang, Y., Tokarev, A.A., Chen, S.H., Cox, R., Andrejic, J., Lipatova, Z., Sciorra, V.A., Emr, S.D., and Segev, N. (2006). TRAPPII subunits are required for the specificity switch of a Ypt-Rab GEF. Nature Cell Biology 8, 1263-1269. 
Nazarko, T.Y., Huang, J., Nicaud, J.-M., Klionsky, D.J., and Sibirny, A.A. (2005). Trs85 is Required for Macroautophagy, Pexophagy and Cytoplasm to Vacuole Targeting in Yarrowia lipolytica and Saccharomyces cerevisiae. Autophagy 1, 37-45.

Pettersen, E.F., Goddard, T.D., Huang, C.C., Couch, G.S., Greenblatt, D.M., Meng, E.C., and Ferrin, T.E. (2004). UCSF Chimera-A visualization system for exploratory research and analysis. Journal of Computational Chemistry 25, 1605-1612.

Punjani, A., Rubinstein, J.L., Fleet, D.J., and Brubaker, M.A. (2017). cryoSPARC: algorithms for rapid unsupervised cryo-EM structure determination. Nature Methods 14, 290-296.

Pylypenko, O., Rak, A., Durek, T., Kushnir, S., Dursina, B.E., Thomae, N.H., Constantinescu, A.T., Brunsveld, L., Watzke, A., Waldmann, H., et al. (2006). Structure of doubly prenylated Ypt1:GDI complex and the mechanism of GDI-mediated Rab recycling. EMBO J 25, 13-23.

Rak, A., Pylypenko, O., Durek, T., Watzke, A., Kushnir, S., Brunsveld, L., Waldmann, H., Goody, R.S., and Alexandrov, K. (2003). Structure of Rab GDP-Dissociation Inhibitor in Complex with Prenylated YPT1 GTPase. Science 302, 646-650.

Riedel, F., Galindo, A., Muschalik, N., and Munro, S. (2018). The two TRAPP complexes of metazoans have distinct roles and act on different Rab GTPases. J Cell Biol 217, 601-617.

Robinson, J.S., Klionsky, D.J., Banta, L.M., and Emr, S.D. (1988). Protein sorting in Saccharomyces cerevisiae: isolation of mutants defective in the delivery and processing of multiple vacuolar hydrolases. Mol Cell Biol 8, 4936-4948.

Sacher, M., Jiang, Y., Barrowman, J., Scarpa, A., Burston, J., Zhang, L., Schieltz, D., Yates, J.R., Abeliovich, H., and Ferro-Novick, S. (1998). TRAPP, a highly conserved novel complex on the cis-Golgi that mediates vesicle docking and fusion. EMBO J 17, 2494-2503.

Sacher, M., Barrowman, J., Schieltz, D., Iii, J.R.Y., and Ferro-Novick, S. (2000). Identification and characterization of five new subunits of TRAPP. European Journal of Cell Biology 10.

Sacher, M., Barrowman, J., Wang, W., Horecka, J., Zhang, Y., Pypaert, M., and Ferro-Novick, S. (2001). TRAPP I Implicated in the Specificity of Tethering in ER-to-Golgi Transport. Molecular Cell 7, 433-442.

Sacher, M., Shahrzad, N., Kamel, H., and Milev, M.P. (2019). TRAPPopathies: An emerging set of disorders linked to variations in the genes encoding transport protein particle (TRAPP)associated proteins. Traffic 20, 5-26.

Scheres, S.H.W. (2012). RELION: Implementation of a Bayesian approach to cryo-EM structure determination. Journal of Structural Biology 180, 519-530.

Segev, N. (1991). Mediation of the Attachment or Fusion Step in Vesicular Transport by the GTP-Binding Ypt1 Protein. Science 252, 1553. 
Segev, N., Mulholland, J., and Botstein, D. (1988). The yeast GTP-binding YPT1 protein and a mammalian counterpart are associated with the secretion machinery. Cell 52, 915-924.

Sikorski, R.S., and Hieter, P. (1989). A System of Shuttle Vectors and Yeast Host Strains Designed for Efficient Manipulation of DNA in Saccharomyces Cerevisiae. Genetics 122, 1927.

Stanga, D., Zhao, Q., Milev, M.P., Saint-Dic, D., Jimenez-Mallebrera, C., and Sacher, M. (2019). TRAPPC11 functions in autophagy by recruiting ATG2B-WIPI4/WDR45 to preautophagosomal membranes. Traffic 20, 325-345.

Suvorova, E.S., Duden, R., and Lupashin, V.V. (2002). The Sec34/Sec35p complex, a Ypt1p effector required for retrograde intra-Golgi trafficking, interacts with Golgi SNAREs and COPI vesicle coat proteins. J Cell Biol 157, 631-643.

Tan, D., Cai, Y., Wang, J., Zhang, J., Menon, S., Chou, H.-T., Ferro-Novick, S., Reinisch, K.M., and Walz, T. (2013). The EM structure of the TRAPPIII complex leads to the identification of a requirement for COPII vesicles on the macroautophagy pathway. Proc Natl Acad Sci U S A 110, 19432-19437.

Tan, Y.Z., Baldwin, P.R., Davis, J.H., Williamson, J.R., Potter, C.S., Carragher, B., and Lyumkis, D. (2017). Addressing Preferred Specimen Orientation in Single-Particle Cryo-EM through Tilting. Nat Methods 14, 793-796.

Taussig, D., Lipatova, Z., and Segev, N. (2014). Trs20 is required for TRAPP III complex assembly at the PAS and its function in autophagy. Traffic 15, 327-337.

Terwilliger, T.C., Ludtke, S.J., Read, R.J., Adams, P.D., and Afonine, P.V. (2020). Improvement of cryo-EM maps by density modification. Nature Methods 17, 923-927.

Thomas, L.L., and Fromme, J.C. (2016). GTPase cross talk regulates TRAPPII activation of Rab11 homologues during vesicle biogenesis. J Cell Biol 215, 499-513.

Thomas, L.L., Joiner, A.M.N., and Fromme, J.C. (2018). The TRAPPIII complex activates the GTPase Ypt1 (Rab1) in the secretory pathway. J Cell Biol 217, 283-298.

Thomas, L.L., van der Vegt, S.A., and Fromme, J.C. (2019). A Steric Gating Mechanism Dictates the Substrate Specificity of a Rab-GEF. Developmental Cell 48, 100-114.e9.

Turnbull, A.P., Kümmel, D., Prinz, B., Holz, C., Schultchen, J., Lang, C., Niesen, F.H., Hofmann, K.-P., Delbrück, H., Behlke, J., et al. (2005). Structure of palmitoylated BET3: insights into TRAPP complex assembly and membrane localization. EMBO J 24, 875-884.

Wang, W., Sacher, M., and Ferro-Novick, S. (2000). Trapp Stimulates Guanine Nucleotide Exchange on Ypt1p. J Cell Biol 151, 289-296. 
Wu, Y.-W., Oesterlin, L.K., Tan, K.-T., Waldmann, H., Alexandrov, K., and Goody, R.S. (2010). Membrane targeting mechanism of Rab GTPases elucidated by semisynthetic protein probes. Nature Chemical Biology 6, 534-540.

Yamakawa, H., Seog, D.-H., Yoda, K., Yamasaki, M., and Wakabayashi, T. (1996). Uso1 Protein Is a Dimer with Two Globular Heads and a Long Coiled-Coil Tail. Journal of Structural Biology 116, 356-365.

Yang, J., Anishchenko, I., Park, H., Peng, Z., Ovchinnikov, S., and Baker, D. (2020). Improved protein structure prediction using predicted interresidue orientations. PNAS 117, 1496-1503.

Yugandhar, K., Wang, T.-Y., Leung, A.K.-Y., Lanz, M.C., Motorykin, I., Liang, J., Shayhidin, E.E., Smolka, M.B., Zhang, S., and Yu, H. (2020). MaXLinker: Proteome-wide Cross-link Identifications with High Specificity and Sensitivity. Molecular \& Cellular Proteomics 19, 554568.

Zhang, K. (2016). Gctf: Real-time CTF determination and correction. Journal of Structural Biology 193, 1-12.

Zhang, Z., Bai, M., Barbosa, G.O., Chen, A., Wei, Y., Luo, S., Wang, X., Wang, B., Tsukui, T., Li, H., et al. (2020). Broadly conserved roles of TMEM131 family proteins in intracellular collagen assembly and secretory cargo trafficking. Science Advances 6, eaay 7667.

Zhao, S., Li, C.M., Luo, X.M., Siu, G.K.Y., Gan, W.J., Zhang, L., Wu, W.K.K., Chan, H.C., and Yu, S. (2017). Mammalian TRAPPIII Complex positively modulates the recruitment of Sec13/31 onto COPII vesicles. Sci Rep 7.

Zheng, S.Q., Palovcak, E., Armache, J.-P., Verba, K.A., Cheng, Y., and Agard, D.A. (2017). MotionCor2: anisotropic correction of beam-induced motion for improved cryo-electron microscopy. Nature Methods 14, 331-332.

Zivanov, J., Nakane, T., Forsberg, B.O., Kimanius, D., Hagen, W.J., Lindahl, E., and Scheres, S.H. (2018). New tools for automated high-resolution cryo-EM structure determination in RELION-3. ELife 7, e42166.

Zivanov, J., Nakane, T., and Scheres, S.H.W. (2020). Estimation of high-order aberrations and anisotropic magnification from cryo-EM data sets in RELION-3.1. IUCrJ 7, 253-267.

Zong, M., Wu, X., Chan, C.W.L., Choi, M.Y., Chan, H.C., Tanner, J.A., and Yu, S. (2011). The Adaptor Function of TRAPPC2 in Mammalian TRAPPs Explains TRAPPC2-Associated SEDT and TRAPPC9-Associated Congenital Intellectual Disability. PLoS One 6.

Zoppino, F.C.M., Militello, R.D., Slavin, I., Álvarez, C., and Colombo, M.I. (2010). Autophagosome Formation Depends on the Small GTPase Rab1 and Functional ER Exit Sites. Traffic 11, 1246-1261. 
Figure 2
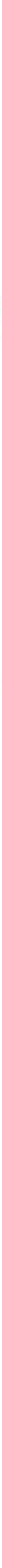

D

Percent of total cross-links

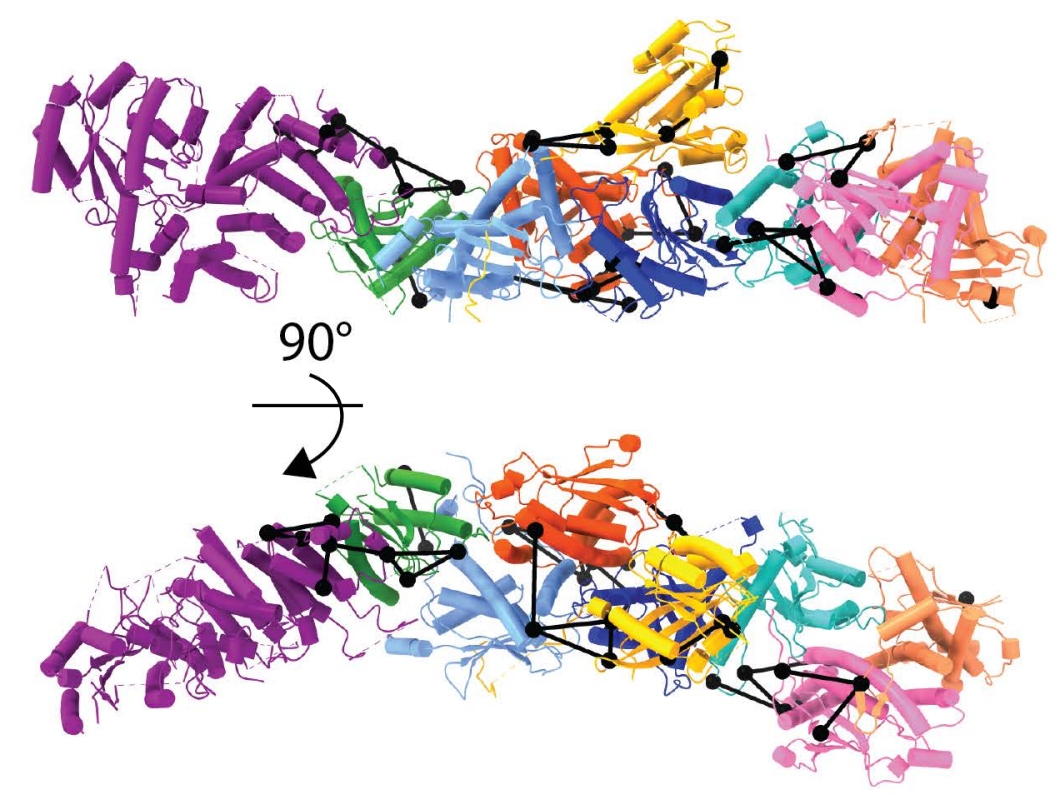

E Distribution of mapped cross-links
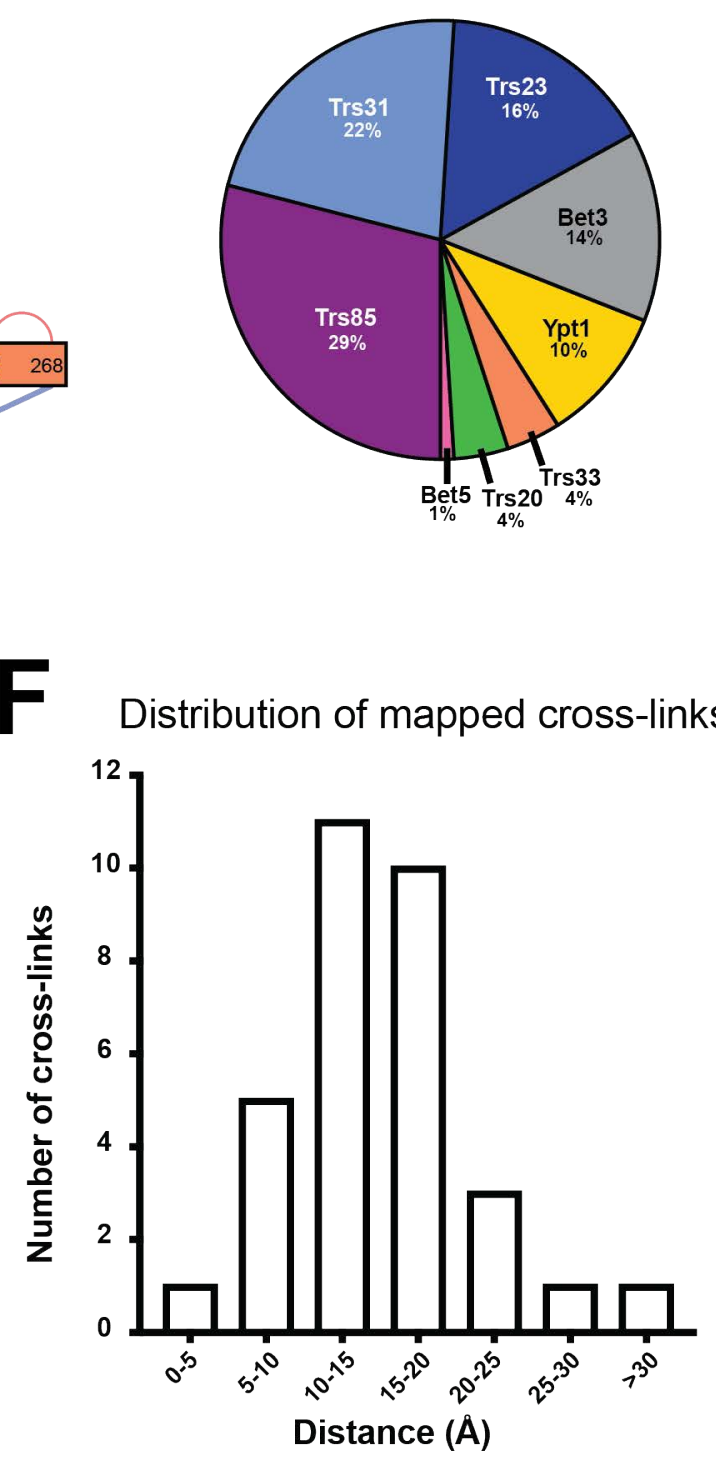

Distance (A) 

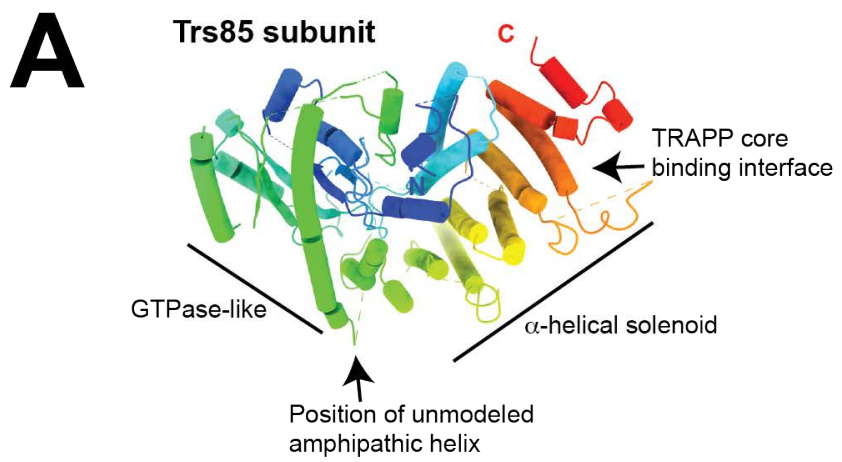

\section{Across model organisms}

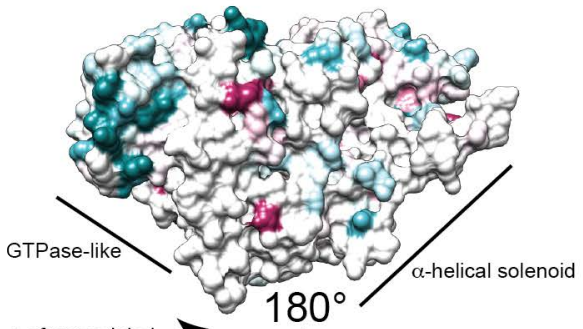

Position of unmodeled $\rightarrow$ amphipathic helix
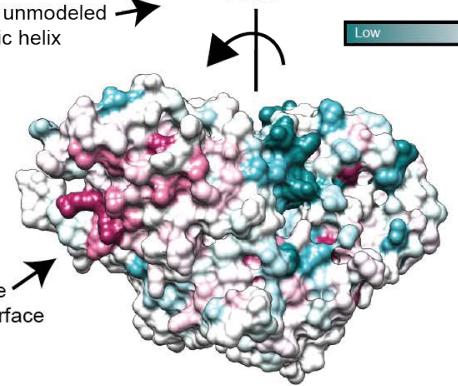

TRAPP core binding interface
Among closely related species

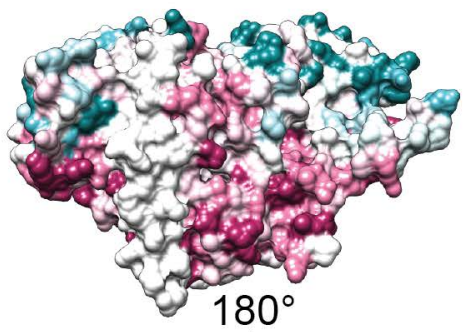

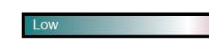

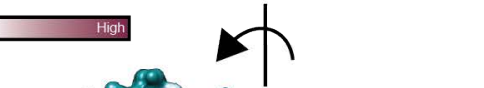



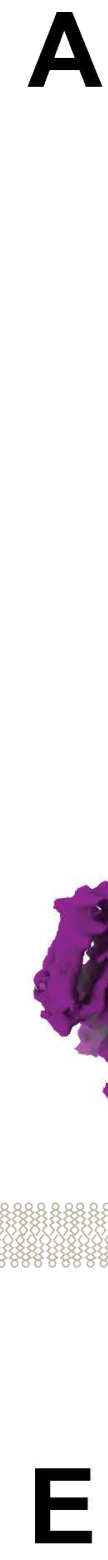

E
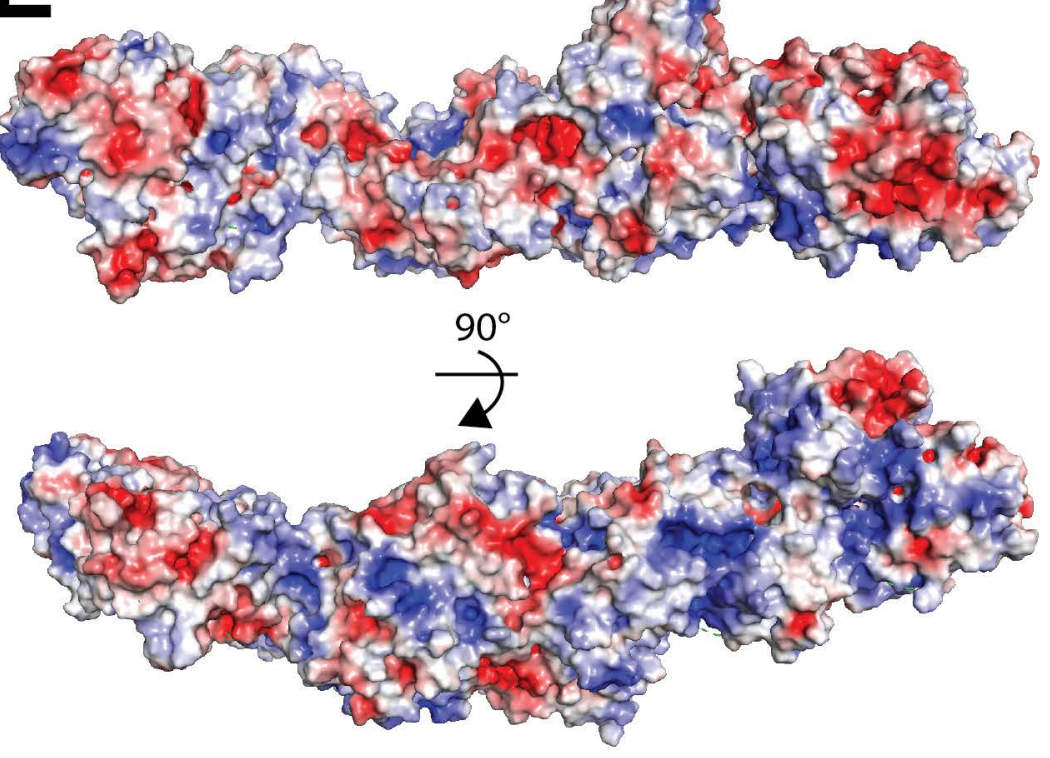

Negative Positive

C-terminus of Ypt1 HVD
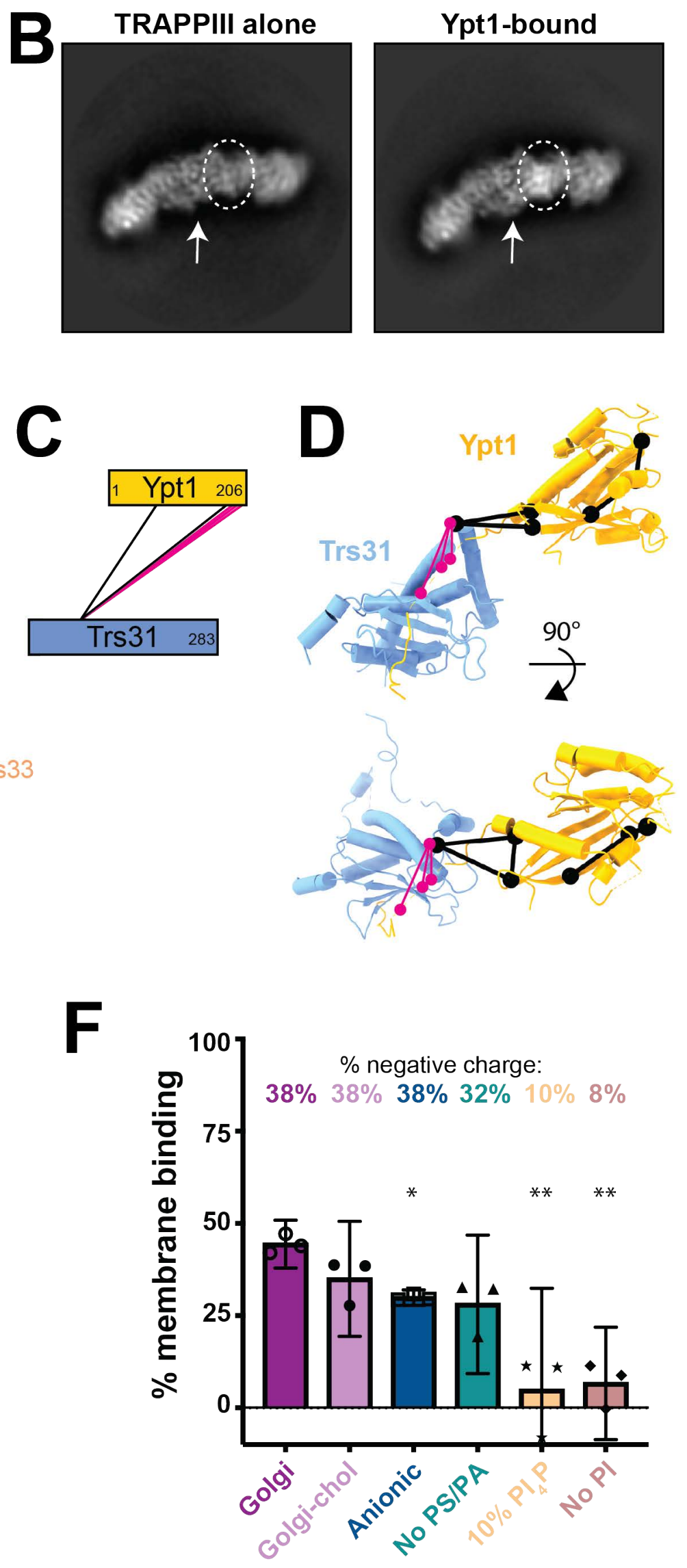

Type of liposomes 
Figure 6 bioRxiv preprindoi: https://doi.org/10.1101/2020.10.08.332312; this version posted October 8, 2020. The copyright holder for this preprint (which was not $\&$ tified by peer review) is the author/funder, who has granted bioRxiv a license to display the preprint in perpetuity. It is made Trs85
available undefaCG-BYHC-ND 4.0 International license.
Bet3-A

\section{0}

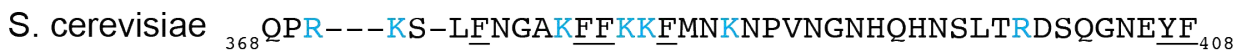
C. elegans ${ }_{337}$ QKKGIGKSF-TSMKRWFGSGTAL-----------SNMATPIT $\underline{Y A}_{368}$ D. melanogaster ${ }_{386}$ NKKGVSKSLLSATKRWFVTSKPG---------AG-ANNQNAVIYTT $_{415}$ D. rario ${ }_{402}$ SRKGLSRSLFSATKKLFGGGKVPEKSI-----AE-LKNTAGLLYPP $_{440}$ X. tropicalis ${ }_{404}$ SRKGLSRSLLSATKKWFSGSKVPEKSI-----NE-LKNTSGLLYPP $_{442}$ M. musculus ${ }_{377}$ SRKGLSRSLFSATKKWFSGSKVPEKSI-----NE-LKNTSGLLY ${ }_{415}$ H. sapiens ${ }_{377}$ SRKGLSRSLFSATKKWFSGSKVPEKSI-----ND-LKNTSGLLY P ${ }_{415}$

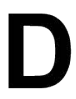

WT 378 KFFKKFMNK $_{386}$ 4A 2 (1) 378 AFFAFMNA ${ }_{386}$

7A 2I글 378 AAAAAAMNA $_{386}$ 4E 2 (3) 378 EFFEEFMNE ${ }_{386}$
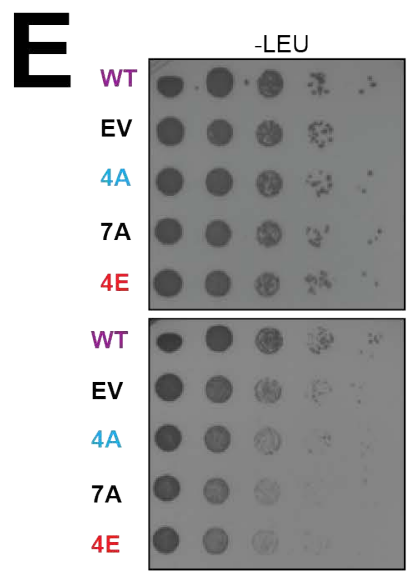
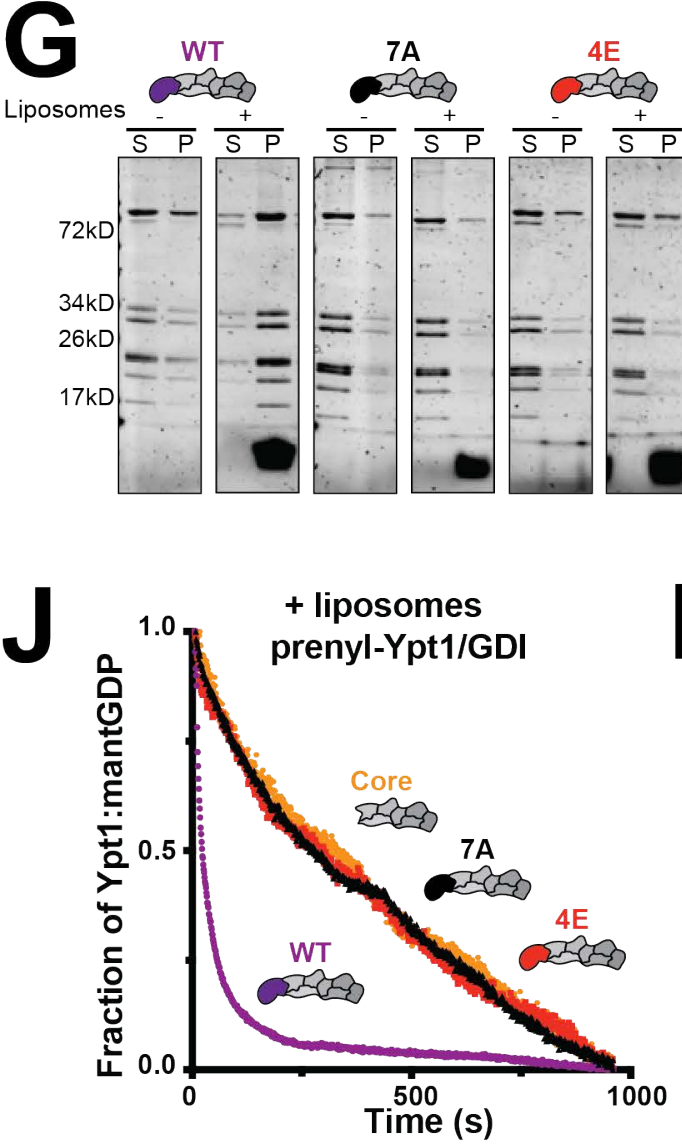
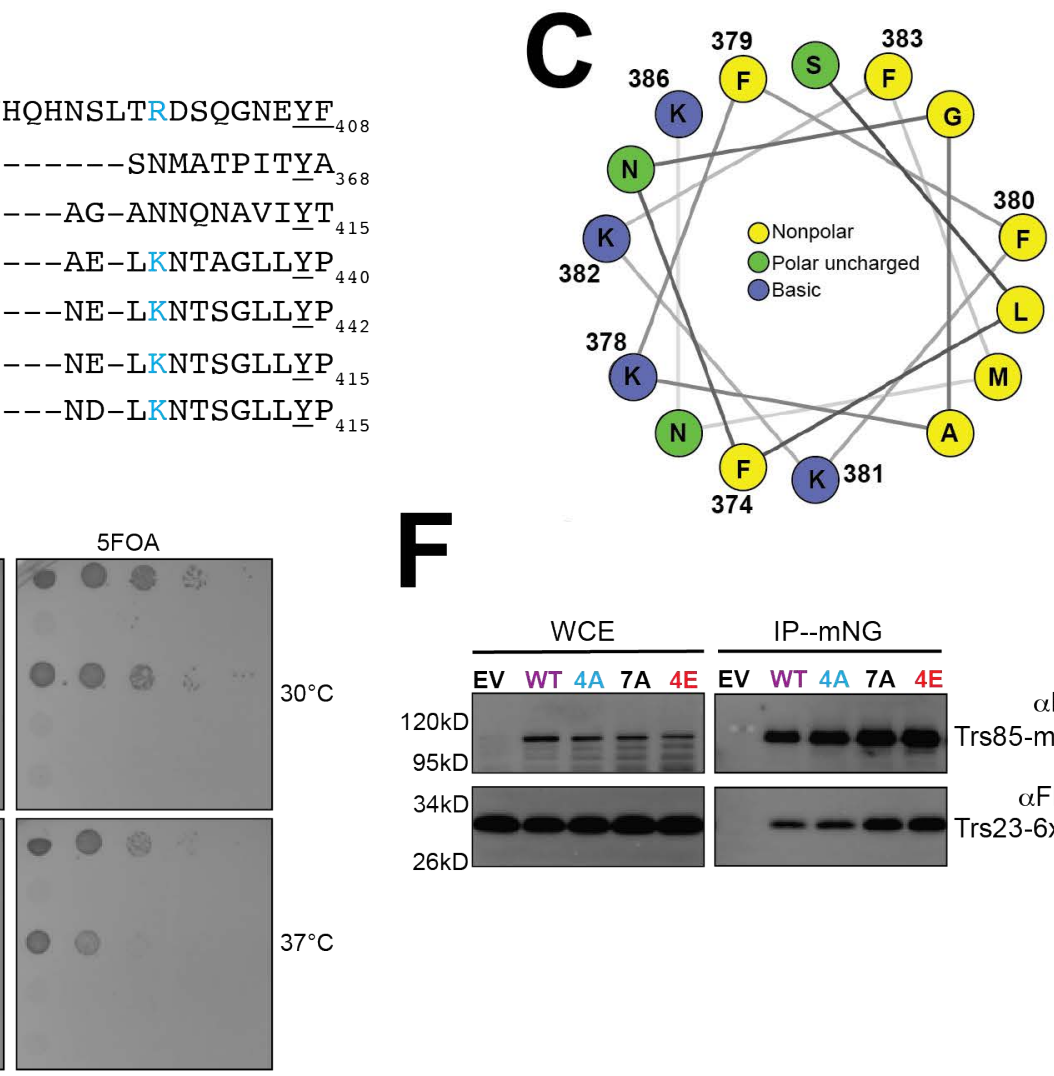

F
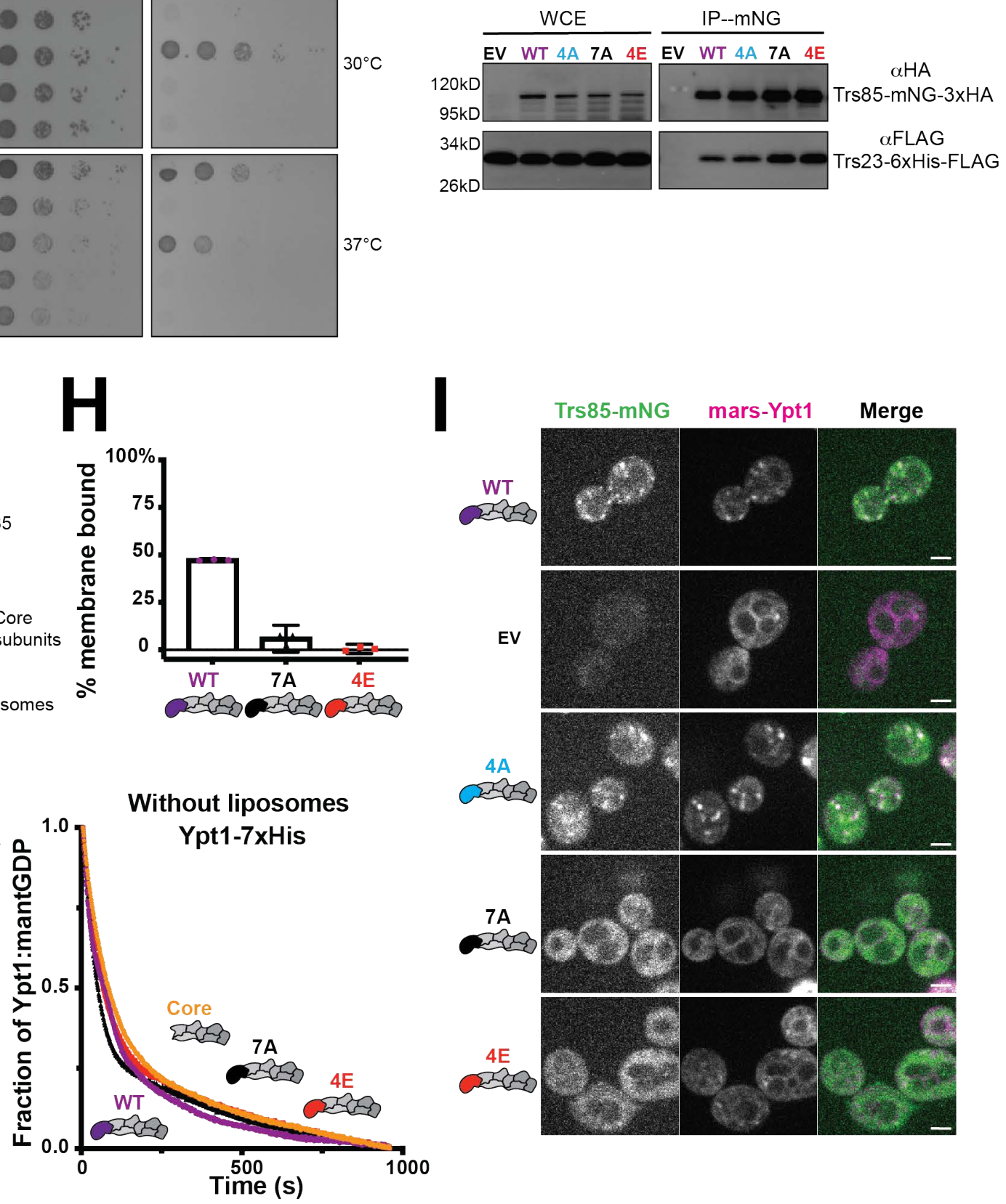
Figure 7

A

Core binding mutations

Membrane binding mutations

K378A,

F379A

F380A,

R618E, R618A,

R619E, R619A,

R620E, R620A,

K378A, K381A, K378E,

K381A, K382A, K381E,

K382A, F383A, K382E,

$\frac{\text { R621E }}{+} \frac{\text { R621A }}{+} \frac{501-698}{+}$

$\frac{\mathrm{EV}}{-+} \frac{\mathrm{WT}}{-+} \frac{\mathrm{K} 386 \mathrm{~A}}{-+} \frac{\mathrm{K} 386 \mathrm{~A}}{-+} \frac{\mathrm{K} 386 \mathrm{E}}{-+}$
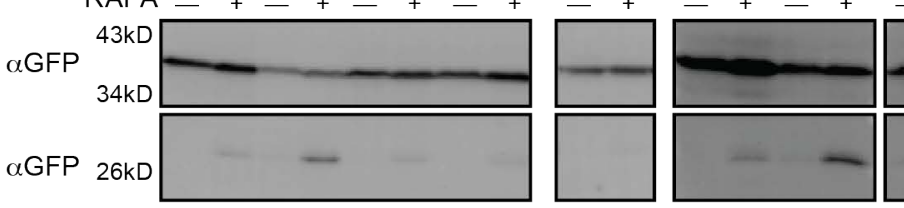

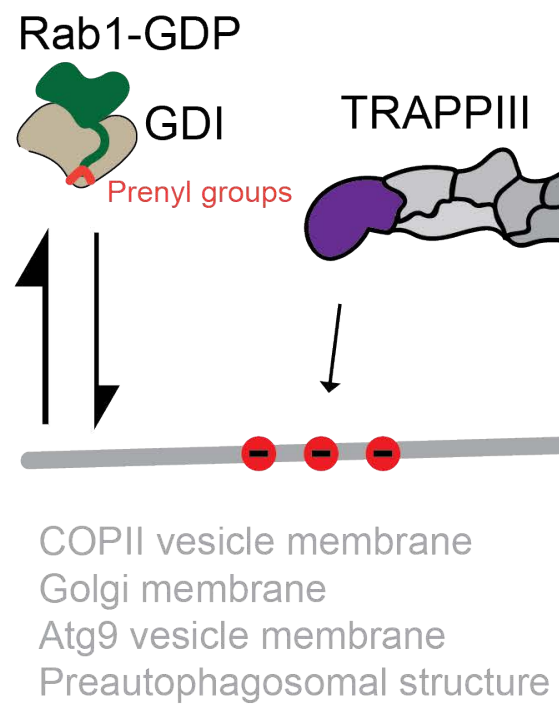

GFP-Atg8

GFP

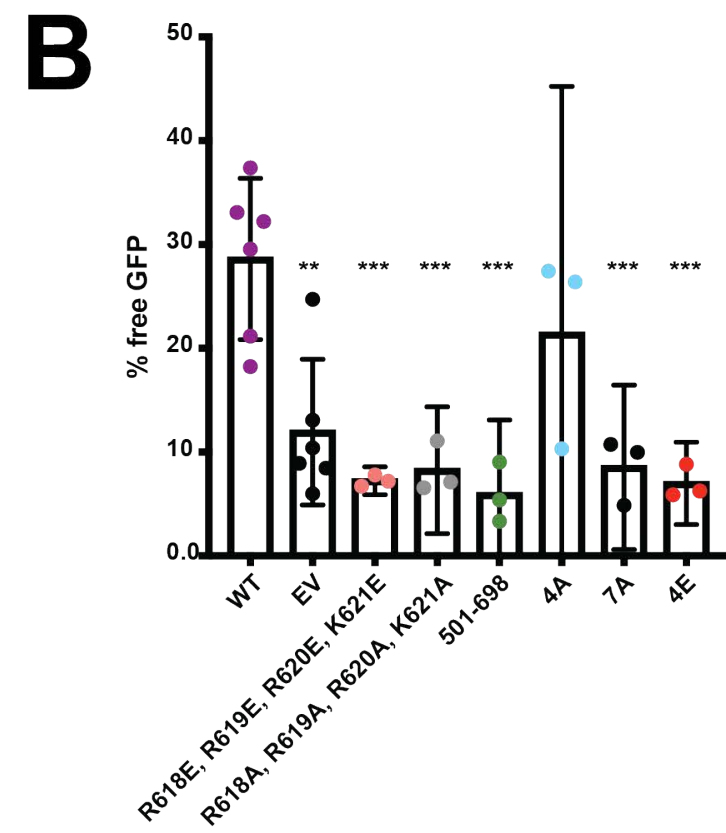

Rab1-GTP
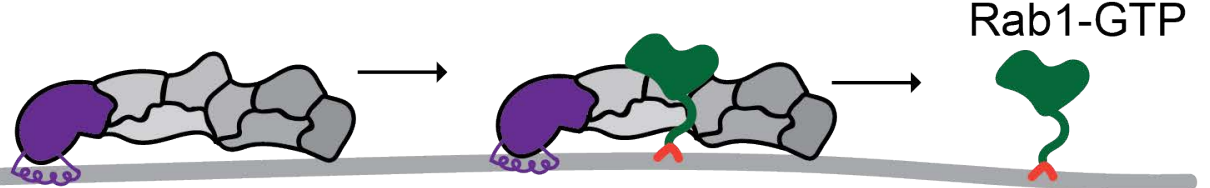
Figure S1

A
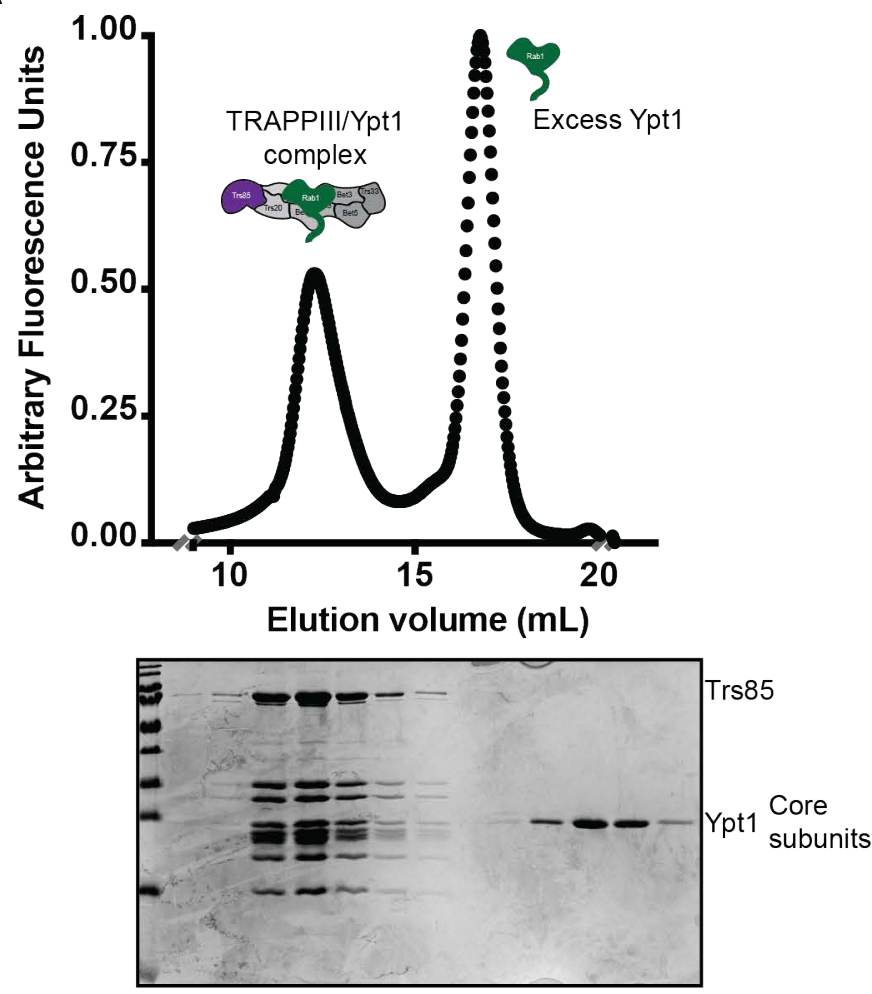

B

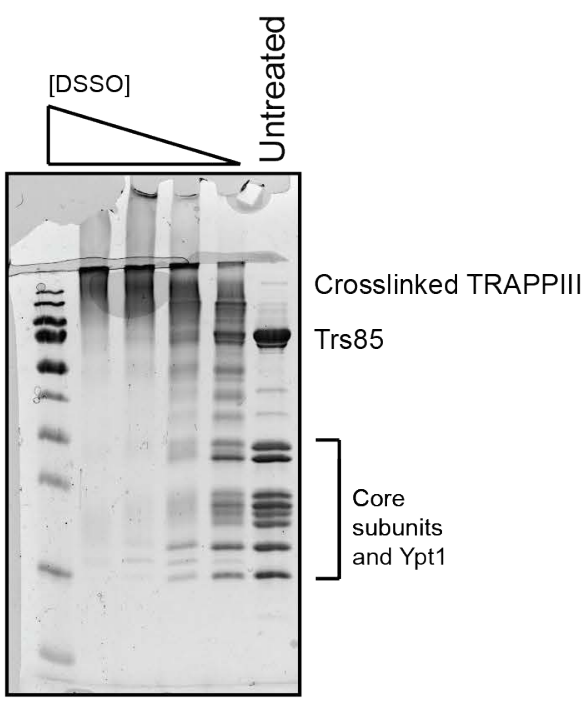


Figure S2 (which was not certified by peer review) is the author/funder, who has granted bioRxiv a license to display the preprint in perpetuity. It is made available under aCC-BY-NC-ND 4.0 International license.

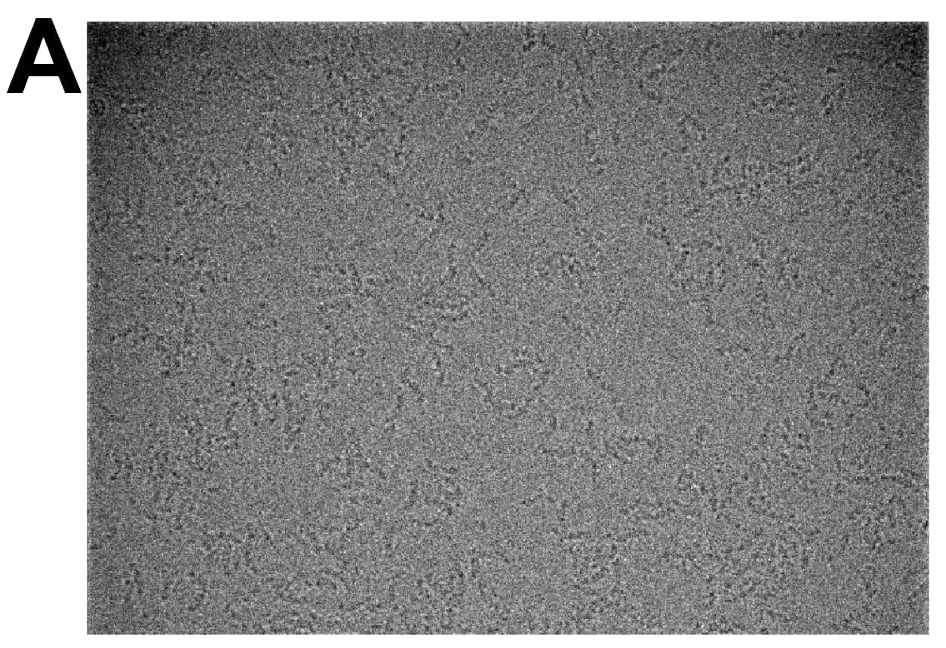

\section{B}

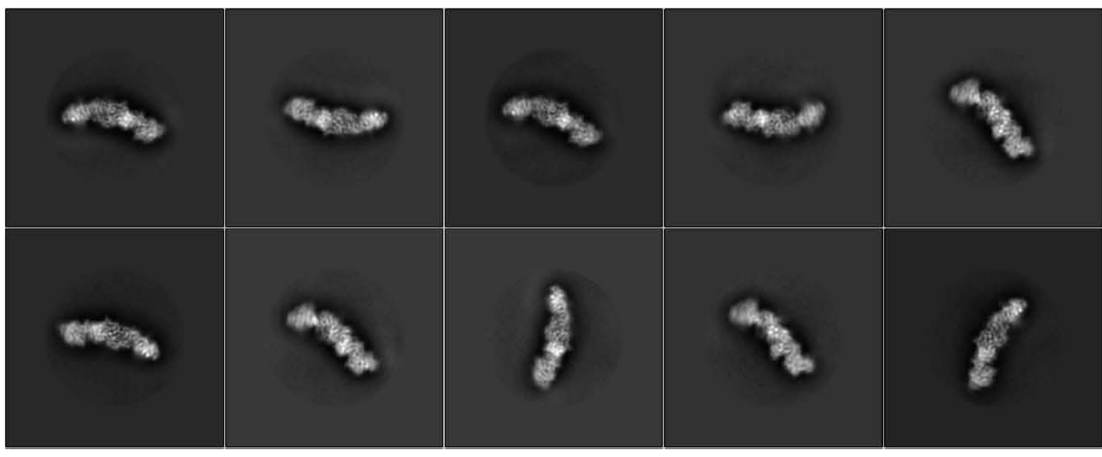

D
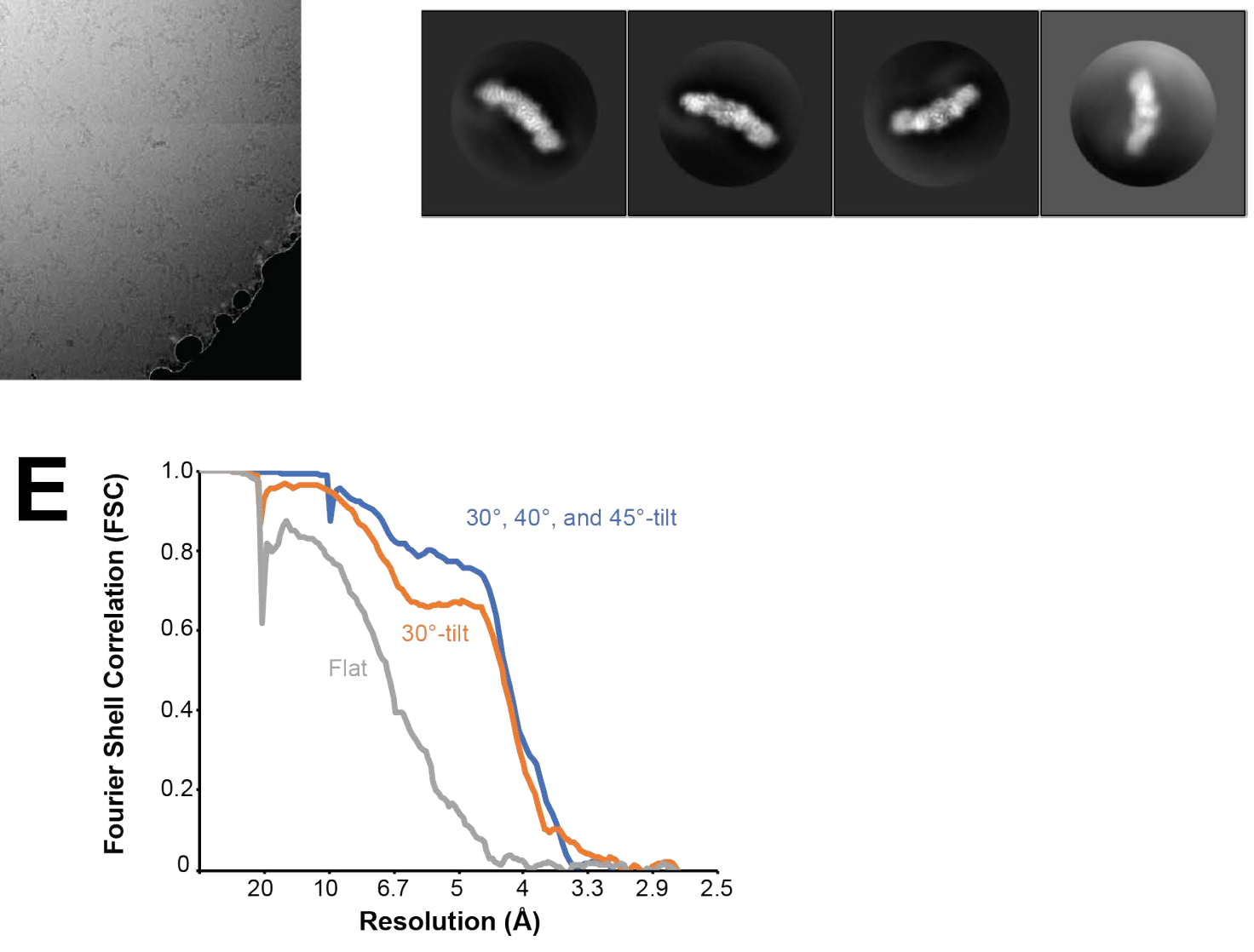

\section{Orientation Distributions}

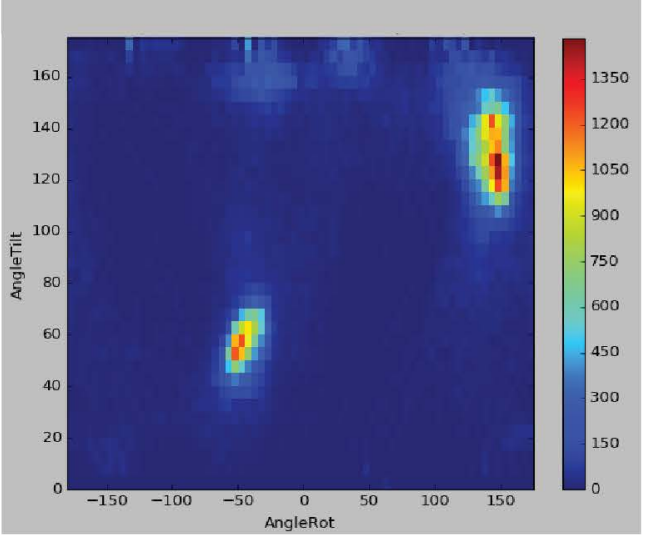

Flat

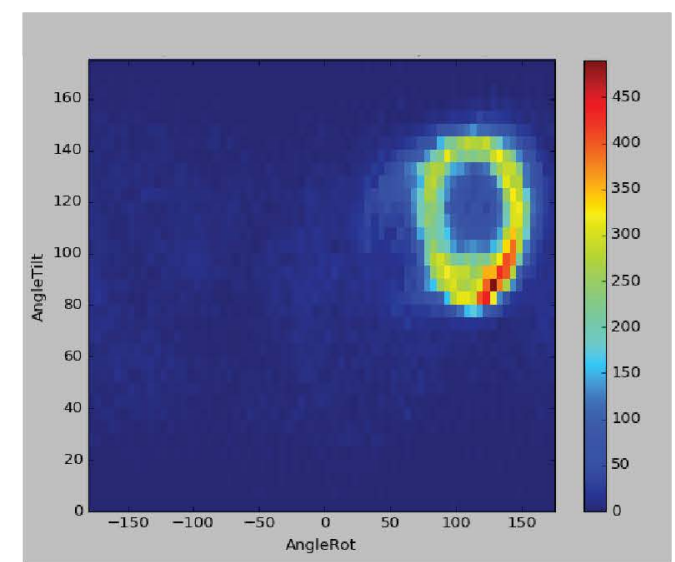

$30^{\circ}$ tilted data

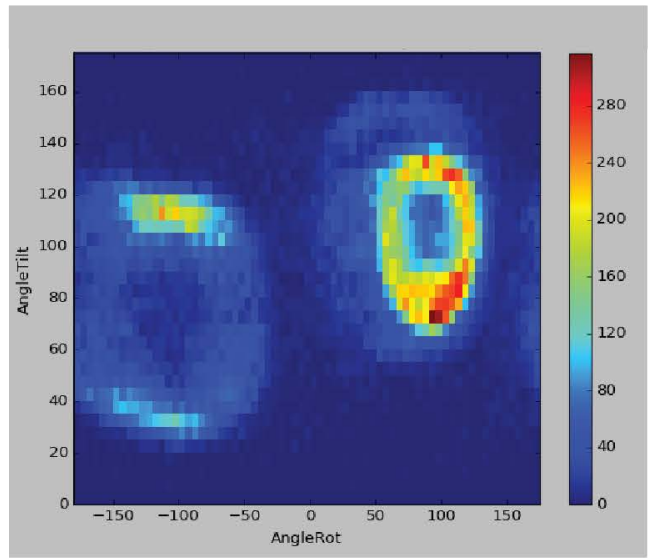

$30^{\circ}, 40^{\circ}$, and $45^{\circ}$ tilted data 
Figure S3 bioRxiv preprint doi: https://doi.org/10.1101/2020.10.08.332312; this version posted October 8, 2020. The copyright holder for this preprint (which was not certified by peer review) is the author/funder, who has granted bioRxiv a license to display the preprint in perpetuity. It is made

Cornell Arctica $40^{\circ}$ and $45^{\circ}$ tilt data available under aCC-BY-NC-ND 4.0 International license.

\section{K3 detector \\ 5012 movies}

$45^{\circ}$-tilt: $\sim 215 \mathrm{~K}$ particles

$40^{\circ}$-tilt: $\sim 316 \mathrm{~K}$ particles

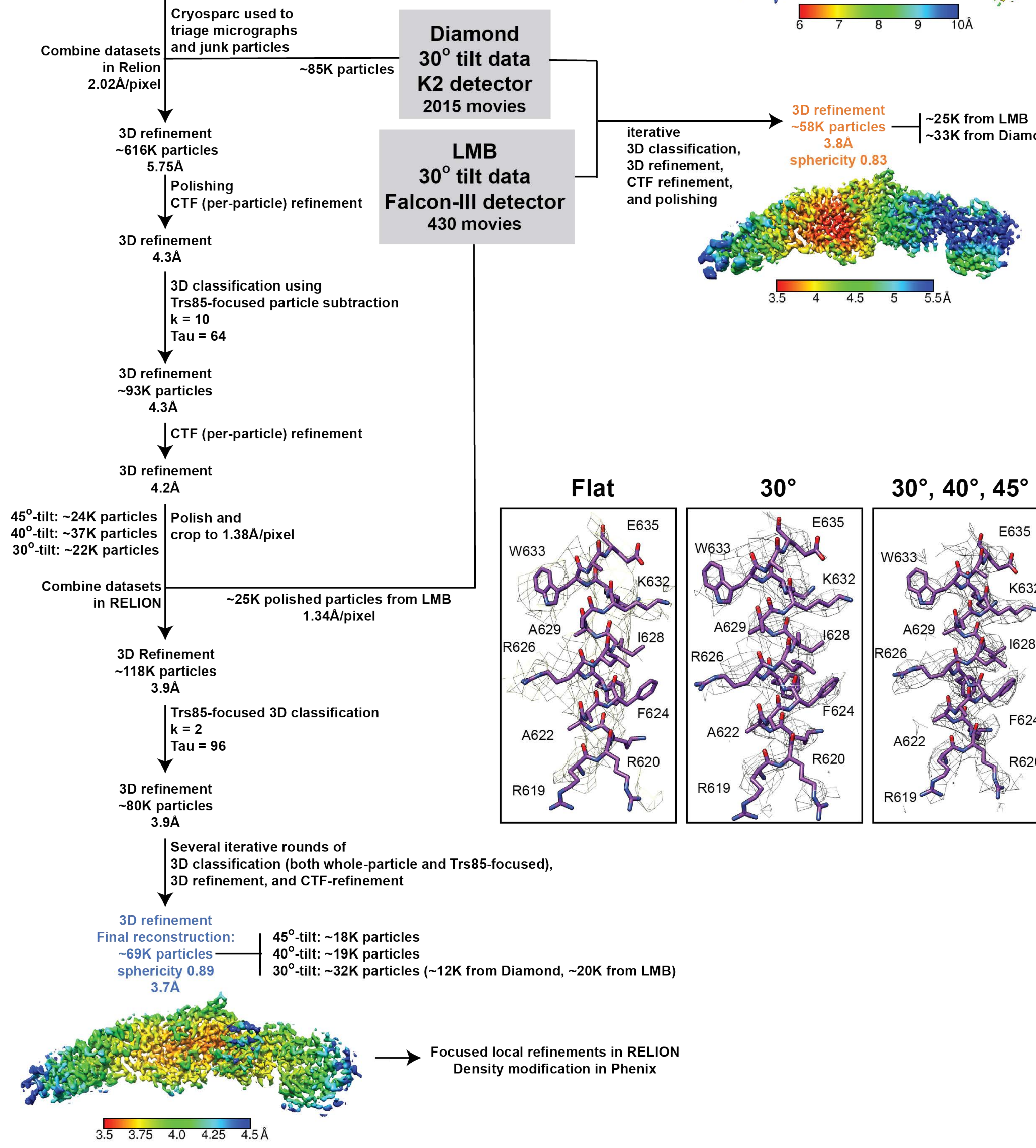
Cryosparc used to
triage micrographs Combine datasets and junk particles in Relion $2.02 A / p i x e l$

3D refinement

$\sim 616 \mathrm{~K}$ particles

5.75A

Polishing

CTF (per-particle) refinement

$3 D$ refinement

4.3A

Trs85-focused particle subtraction

$k=10$

Tau $=64$

3D refinement

$\sim 93 \mathrm{~K}$ particles

4.3A

$\downarrow$ CTF (per-particle) refinement

3D refinement

$4.2 A$

$45^{\circ}$-tilt: $\sim 24 \mathrm{~K}$ particles

$40^{\circ}$-tilt: $\sim 37 \mathrm{~K}$ particles

$30^{\circ}$-tilt: $\sim 22 \mathrm{~K}$ particles

Combine datasets in RELION

Polish and crop to $1.38 \mathrm{~A} / \mathrm{pixel}$

25K polished particles from LMB 1.34A/pixel

3D Refinement

118K particles

$3.9 A$

Trs85-focused 3D classification

$k=2$

Tau $=96$

3D refinement

$\sim 80 \mathrm{~K}$ particles

3.9A

Several iterative rounds of

3D classification (both whole-particle and Trs85-focused),

$\downarrow$ 3D refinement, and CTF-refinement

3D refinement

Final reconstruction:

$\sim 69 \mathrm{~K}$ particles sphericity 0.89

$45^{\circ}$-tilt: $\sim 18 \mathrm{~K}$ particles

$40^{\circ}$-tilt: $\sim 19 \mathrm{~K}$ particles

$30^{\circ}$-tilt: $\sim 32 \mathrm{~K}$ particles $(\sim 12 \mathrm{~K}$ from Diamond, $\sim 20 \mathrm{~K}$ from $\mathrm{LMB})$

$3.7 \AA$

Focused local refinements in RELION Density modification in Phenix

$\longrightarrow \sim 58 \mathrm{~K}$ particles $\longrightarrow \underset{\sim 33 \mathrm{~K} \text { from LMB }}{\sim 25 \mathrm{~K}}$

$\begin{array}{lc}\text { 3D classification, } & 3.8 \mathrm{~A} \\ \text { 3D refinement, } & \text { sphericity } 0.83\end{array}$

CTF refinement, and polishing

Flat

$30^{\circ}$

$30^{\circ}, 40^{\circ}, 45^{\circ}$

$3.5 \quad 3.75 \quad 4.0 \quad 4.25 \quad 4.5 \AA$

LMB

"flat" data

Falcon-III detector 809 movies

$3 \mathrm{D}$ refinement

$\sim 120 \mathrm{~K}$ particles $5.0 \mathrm{~A}$

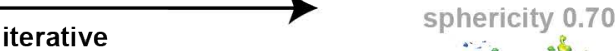

3D classification

and 3D refinement

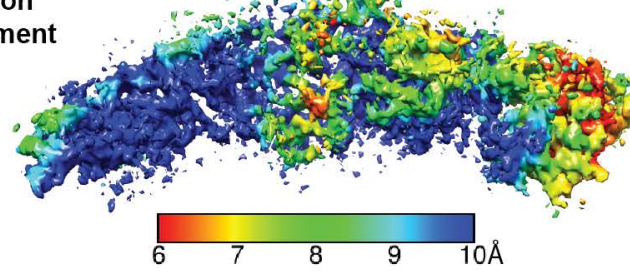


Figure S4 bioRxiv preprint doi: https://doi.org/10.1101/2020.10.08.332312; this version posted October 8, 2020. The copyright holder for this preprint
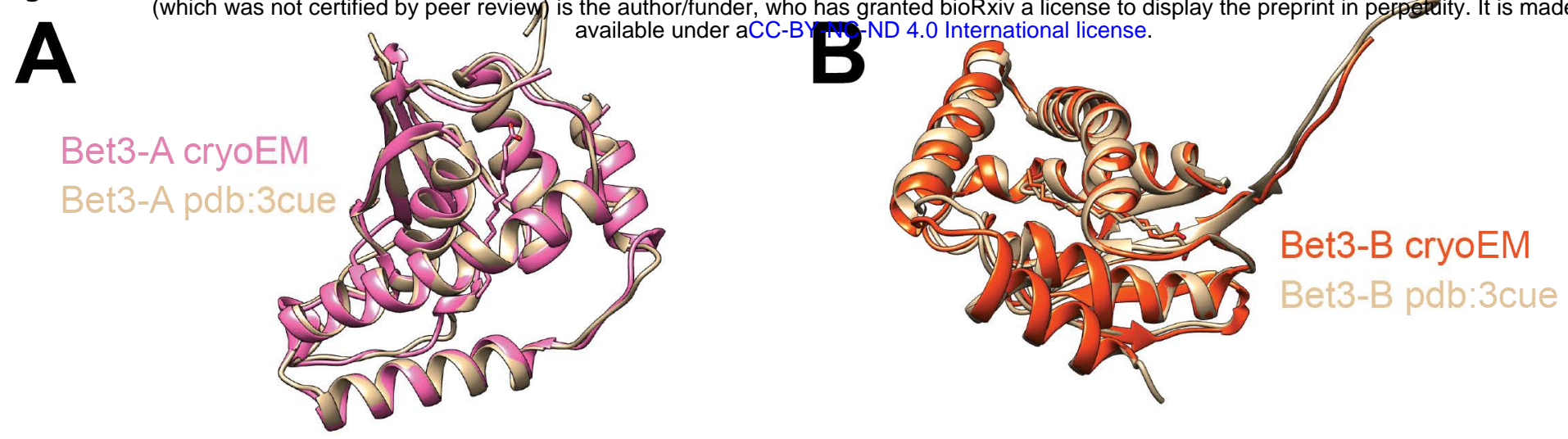

6

Bet5 cryoEM Bet5 pdb:3cue

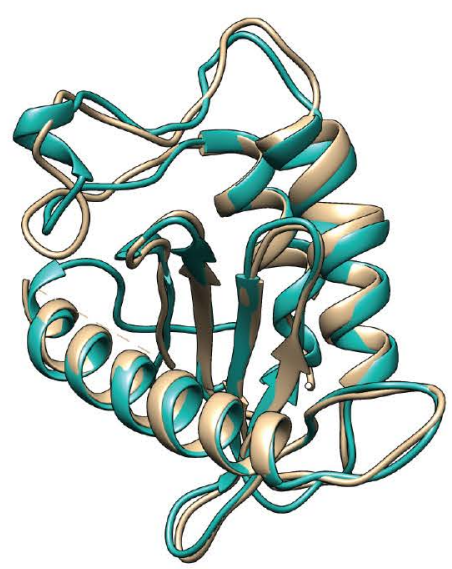

D

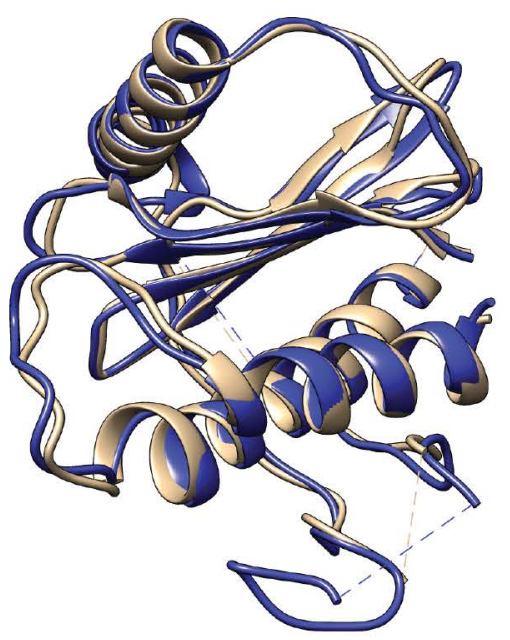

Trs23 cryoEM

E
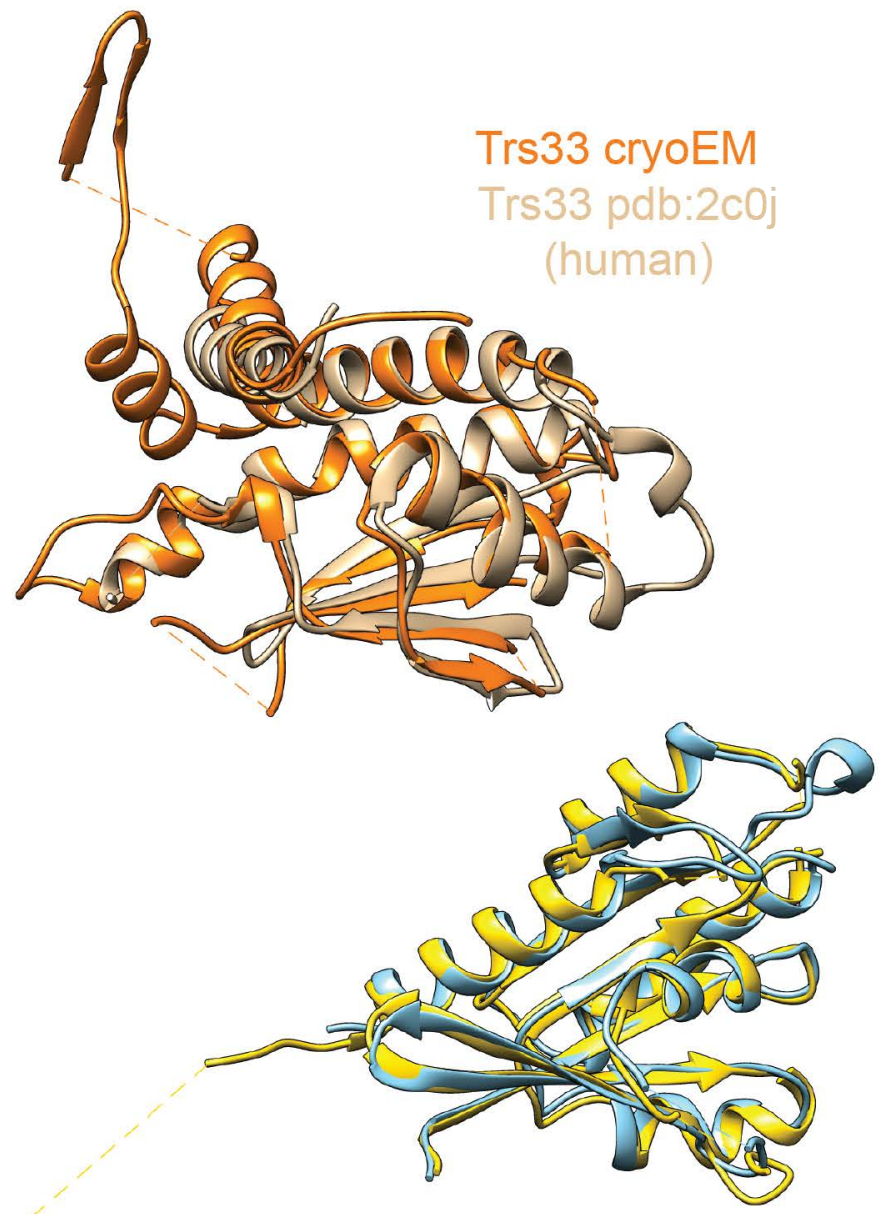

Ypt1 cryoEM Ypt1 pdb:3cue

\section{Trs20 cryoEM} (mouse)

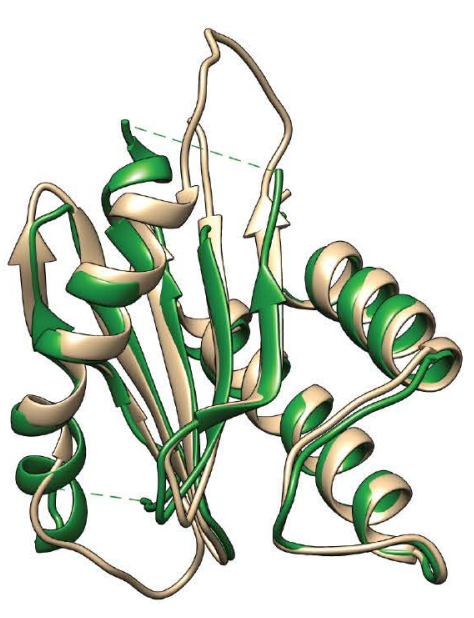

H 